\title{
Opportunities for Ballistic Soft Landing in Binary
}

\author{
Asteroids \\ Onur Çelik ${ }^{1}$ and Joan Pau Sánchez ${ }^{2}$ \\ Space Research Group, Centre of Autonomous and Cyber-Physical Systems, Cranfield University, UK
}

\begin{abstract}
Remote sensing instrumentation on-board missions to asteroids is paramount to address many of the fundamental questions in modern planetary science. Yet, in-situ surface measurements provide the "ground-truth" necessary to validate and enhance the science return of these missions. Nevertheless, due to the dynamical uncertainties associated with the environment near these objects, most missions spend long periods of times stationed afar. Small landers can be used much more daringly, however, and thus have already been identified as valuable assets for insitu exploration. This paper explores the potential for ballistic landing opportunities enabled by the natural dynamics found in binary asteroid systems. The dynamics near a binary asteroid are modelled by means of the Circular Restricted Three Body Problem, which provides a reasonable representation of a standard binary system. Natural landing trajectories are then sought that allow for a deployment from the exterior region and touchdown with minimum local-vertical velocity. The results show that while landing on the main body of the system would require an effective landing system capable to dissipate excess of energy, and avoid bouncing off the asteroid, the smaller companion offers the prospect of simple ballistic landing opportunities.
\end{abstract}

\section{Nomenclature}

$a \quad=$ Semi-major axis of the mutual binary asteroid orbit $[\mathrm{km}]$

${ }^{1}$ Graduate Student, School of Aerospace, Transport and Manufacturing, Cranfield University, UK; now Research Student, Department of Space and Astronautical Sciences, The Graduate University for Advanced Studies (SOKENDAI), Japan.

${ }^{2}$ Lecturer in Space Engineering, School of Aerospace, Transport and Manufacturing, Cranfield University, UK. 


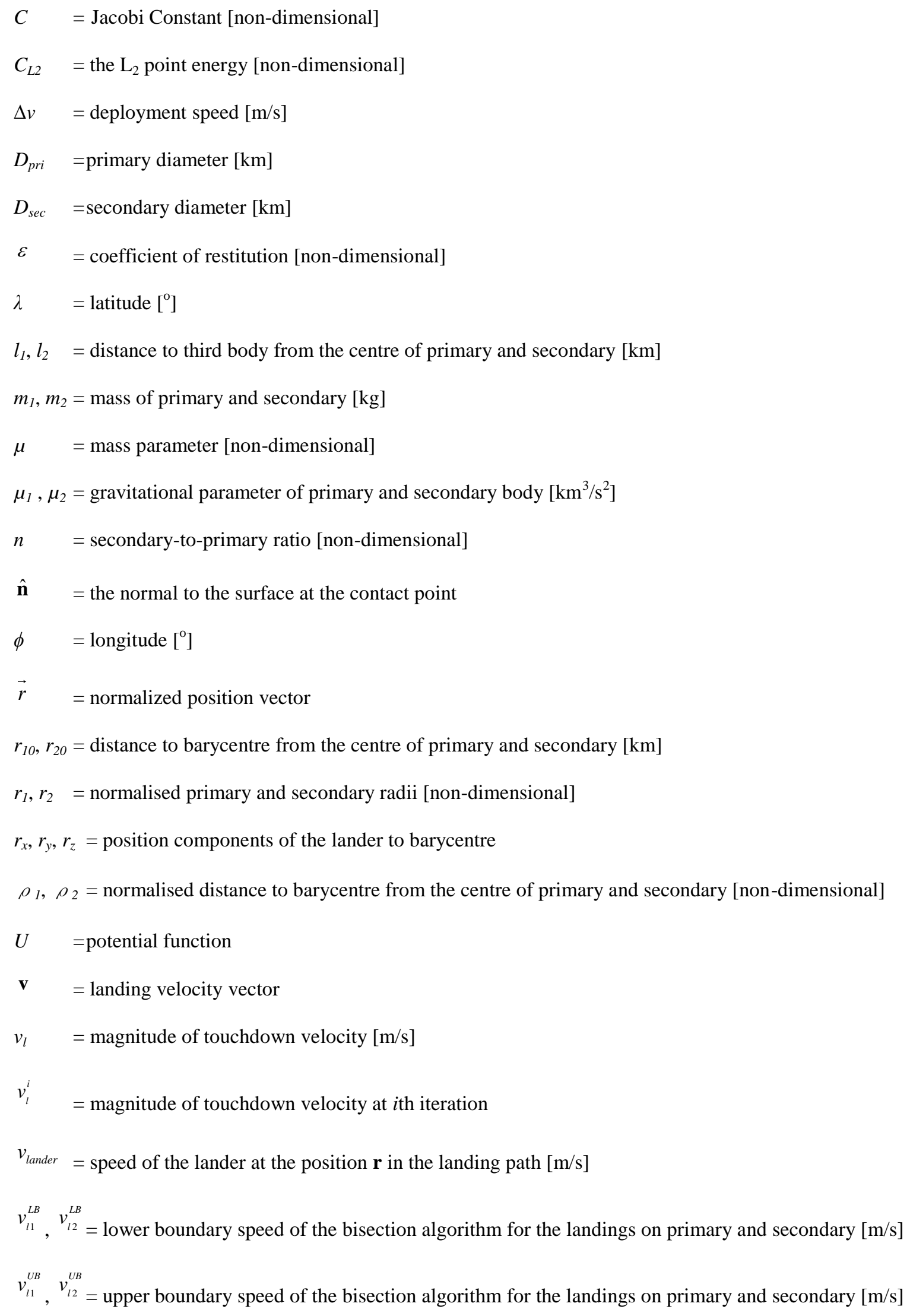


$\mathbf{v}_{L V}^{-}, \mathbf{v}_{L H}^{-}=$local vertical $(\mathrm{LV})$ and local horizontal $(\mathrm{LH})$ velocity vector of the lander an instant before the touchdown

$\mathbf{v}_{L V}^{+}, \mathbf{v}_{L H}^{+}=$local vertical (LV) and local horizontal (LH) velocity vector of the lander an instant after the touchdown

$v_{\text {mother }}=$ orbital speed of the mothership at the position $\mathbf{r}[\mathrm{m} / \mathrm{s}]$

$V \quad=$ normalised speed of third body [non-dimensional]

$x, y, z=$ normalised position components [non-dimensional]

$x_{d}, y_{d}, z_{d}=$ normalised deployment position components [non-dimensional]

$\omega \quad=$ magnitude of angular velocity of the mutual binary orbit $[\mathrm{rad} / \mathrm{sec}]$

$\omega_{1} \quad=$ rotational rate of primary $[\mathrm{rad} / \mathrm{sec}]$

\section{Introduction}

Missions to asteroids have become increasingly attractive in recent years, firstly, because of scientific curiosity, but also due to both their potential impact risk to Earth and prospective economic return. A variety of missions have been proposed, ranging from manned exploration to commercial mining missions. There have also been a number of successful missions to asteroids which brought samples and/or scientific data back to Earth, such as, NEAR-

Shoemaker, Rosetta, Deep Impact, Hayabusa, and Hayabusa-2. Other missions will certainly follow, e.g., OSIRISRex, and will continue improving our knowledge of the formation and evolution of the Solar System, by visiting new asteroids in the future.

Near-Earth Asteroids (NEAs) are among the easiest interplanetary objects that can be reached from the Earth, as well as a potential impact threat to Earth. About $15 \%$ of the NEA population is believed to be a binary system [1]. Their formation mechanism has been of interest for decades now, and an explanation has been attempted by various theories. However, a space mission would certainly provide in-depth knowledge about the formation and evolution of binary systems.

Binary asteroids have also been proposed as ideal platforms to investigate the effectiveness of future asteroid deflection techniques. One of the two spacecraft envisaged for AIDA mission is intended to impact the smaller companion of the binary asteroid Didymos, in order to test the kinetic impact deflection technique [2]. A binary 
system is an ideal testbed for such a technique, since the orbital effects of an impact with the smaller companion of a binary can be observed in a much shorter time scale than otherwise possible in an heliocentric orbit [2].

Further increase on the scientific return of missions to binary asteroids, such as for AIDA, would be achieved by landing a scientific payload on their surface. After the bold and successful attempt of NEAR-Shoemaker to land on Eros in 2001 [3], successor spacecraft to small bodies have been equipped with surface landers for in-situ exploration. As a recent example, Hayabusa-2 spacecraft, launched in 2014, carries three small landers/rovers and a MASCOT Lander designed by DLR/CNES [4]. Although it has not been attempted yet, such landers can also be deployed in binary asteroids onto either of two bodies of the system. Given the fact that navigation within a binary asteroid system poses serious challenges, due to its highly perturbed dynamics and its natural satellite, it might not be the best solution to put a mothership at risk by getting it into the vicinity of the binary in order to deploy a lander. The natural dynamics in binary asteroids could instead be exploited to achieve ballistic landing from a safe distance. In this paper, Circular Restricted Three Body Problem (CR3BP) provides a framework within which natural dynamics can be sought to enable landings of unpowered landers requiring no, or minimum, control capabilities. For instance, NanoSats (or other shoebox-sized spacecraft) are promising candidates to satisfy the exploration demands whereas providing a simple and low-cost solution. Their reliability is increasing in low Earth orbit day by day, and there is no reason to believe that it should not increase also for interplanetary medium via future interplanetary missions. The AIDA mission, for example, is proposed to carry two CubeSats to the binary asteroid Didymos [2], one of which is proposed to land on the smaller companion [5], and the interest continues to grow on nanosat solutions for small body surface exploration [6].

Ballistic landings through natural manifold trajectories on binary asteroids are studied by Scheeres \& Tardivel [7]. The study considers vicinity of the collinear Lagrange points as deployment locations. The landing, in their approach, is defined as the first intersection point of a trajectory on the surface. They concluded that the strategy is proved to be successful [7]. Their following work discussed the strategy for a light lander deployment from L2 point and statistical analysis of uncertainties within the MarcoPolo-R mission studies for the binary asteroid $1996 \mathrm{FG}_{3}$ [8]. The natural manifold deliveries to the surface are also discussed for single asteroid systems. Herrera-Sucarrat et al. [9] used the equilibrium points for fuel-free transfers in Restricted Full Two body Problem (RF2BP), which are equivalent to the Lagrange points in CR3BP [9]. However, their landing strategy requires several manoeuvres before 
touching down the surface [9]. Along the same line, the motion of landers on asteroid surfaces after touchdown has been studied by some researchers within the context of various studies [6, 10-11].

This paper focuses on unpowered landings in binary asteroids by investigating natural minimum energy trajectories which could be found within the dynamics of the system. The binary system is considered as having spherical main and natural satellite bodies, which implies point mass gravity for both. This allows us to obtain a general insight into the problem. The previous body of work focused on particular asteroids; however, here a hypothetical binary asteroid has been created, with the median properties of the well-known binary asteroids which have been observed up to date. Landing trajectories are reverse engineered from the surface of both objects in the hypothetical binary system, by propagating the model backwards in time. A dense grid of latitude-longitude nodes, homogenously distributed over the entire surface of both objects, allows us to obtain a general picture of reachable regions and characteristic features of landing trajectories as a function of target latitude-longitude node.

The paper particularly focuses on searching landing opportunities for soft local-vertical touchdown. These are thus directly applicable solutions for legged-type landers, but also potentially desirable landing geometries for other spacecraft, such as cuboid-shaped NanoSats. This kind of landing strategy offers the computational advantage of defining a single landing vector, or configuration, at each point on the surface of asteroid. The backwards propagation of these landing conditions allows us to provide a complete picture of deployment and landing options, under the assumption of a spherical binary asteroid pair. Most of the previous works focused primarily on deployment strategies from a neighbourhood of the L2 point (e.g., [7-9]). Here, however, all across the binary surface, minimum energy landing trajectories are propagated backwards until they reach regions sufficiently far from the barycentre of the binary to be considered safe locations for a deployment from a notional mothership. This safe region is defined as the exterior realm in CR3BP, i.e. when the L2 neck region is closed. This approach yields a much more general overview of deployment and landing opportunities. Deployment opportunities are studied as a consequence of the propagation from the surface of the asteroid, and interesting features, such as deployment speeds and landing duration, are thus derived as a consequence of touchdown speeds.

Finally, once the complete set of minimum energy landing trajectories is computed, two extra features are investigated. Firstly, the coefficient of restitution, or energy damping, required for these landing conditions to settle onto the surface of the asteroid, is computed. Secondly, a qualitative measure of the sensitivity to errors and perturbations is estimated for all landing trajectories. 


\section{Binary Asteroid Systems}

Binary asteroids are systems of two asteroids orbiting around their common centre of mass. Binary asteroids are relatively abundant within the NEA population; about $15 \%$ of asteroids with a diameter larger than $200 \mathrm{~m}$ are believed to have a secondary companion [1]. Moreover, Pravec \& Harris discuss that the binary population among NEAs is concentrated within diameters smaller than $2 \mathrm{~km}$ [12]. It is generally agreed that binary asteroids form due to the rotational break-up of the original progenitor asteroid. According to recent studies, the rotational break-up is mainly the result of a spin-up process, for which the dominant mechanism is suggested to be the YarkovskyO'Keefe-Radzievski-Paddack (YORP) effect. The YORP hypothesises that, due to irregular shape and uneven illumination conditions, reemitted solar infrared radiation from a small asteroid applies a momentum to it, which consequently accelerates rotation of the body [13]. Once the progenitor body's spin rate increases to a critical level by this effect, where centrifugal force exceeds the combined effects of cohesion and gravity, the asteroid starts shedding mass. In due time, this process evolves into a system of a larger main body, referred thereafter as primary, and a smaller companion, referred as secondary.

Binary primaries are observed to have nearly spherical shapes, whereas secondaries have more elongated shapes [14-16]. Secondaries are not larger than half the diameter of primaries and, in most cases, smaller than that [12]. The most observed NEA binaries have the mass ratio of 0.2 or lower [14]. Predictions for mass density varies between $1.33 \mathrm{~g} / \mathrm{cm}^{3}$ to $3 \mathrm{~g} / \mathrm{cm}^{3}$, which is believed to cover $2 / 3$ of the binary asteroids, according to different sources [12, 15 , 17].

For most of the small binary systems (i.e. $<10 \mathrm{~km}$ ) observed in NEA population, secondaries are tidally locked to their primaries, i.e. secondary spin period is equal to its orbital period around the primary [14]. Semi-major axis of an orbit generally appears to be between 1.5 to 5 primary's radius [13-15]. Secondary's orbit generally has low eccentricity [18].

The only visited binary asteroid so far, Ida-Dactyl system, is a main belt asteroid whose binary nature was discovered in 1993 during the flyby of the Galileo mission on its way to Jupiter. Ida-Dactyl system was the first ever binary asteroid system discovered. Although several other asteroid rendezvous and flybys were performed afterwards and will be performed in near future, namely DeepSpace-1, NEAR-Shoemaker, Hayabusa, Rosetta, Dawn and Hayabusa-2, none of them visited or flew by binary asteroids. 
The Binary Asteroid in-situ Explorer (BASiX) mission is proposed to probe the geophysics of a near-Earth Asteroid (NEA), in the light of some of the theories described above [19]. The binary asteroid 1996 FG3 is selected as the target for the BASiX mission [19]. The newly initiated NASA/ESA joint mission concept, Asteroid Impact \& Deflection Assessment (AIDA) mission, is also proposed to visit a binary asteroid, (65803) Didymos, in 2022, in order to test future asteroid deflection strategies whilst improving our understanding of binary asteroid systems [2].

For the research here, a hypothetical binary asteroid is created. It is aimed that the hypothetical binary should provide a reasonable representation of the known binaries. Fig. 1 shows the distribution of the binary asteroids whose primary and secondary diameters, as well as semi-major axis, are known ${ }^{1}$. Among all the binary objects represented in Fig. 1, only the density of a subset of 17 binaries are known, while for the rest a density of 1600 $\mathrm{kg} / \mathrm{m}^{3}$ is generally assumed in order to estimate the various parameters of binaries, including semi-major axis of secondary orbit. Only the subset of 17 known objects is selected to create our representative hypothetical binary object.

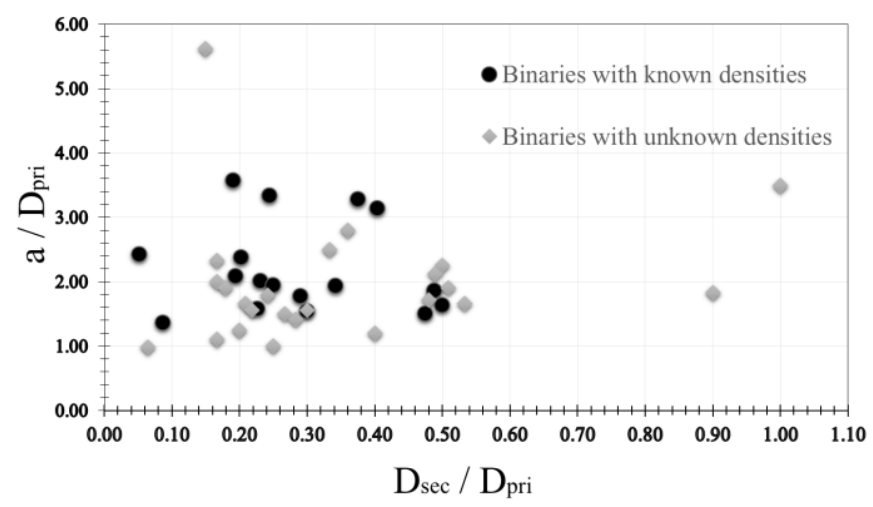

Fig. 1 Secondary radius to primary radius ratio versus semi-major axis to primary radius ratio for observed binary asteroids ${ }^{1}$.

Table 1 shows the mean and median values of the subset of objects with known primary and secondary diameters, semi-major axis of secondary's orbit and mass density, which is generally the density of primary and it is assumed here that both bodies have the same value.

\footnotetext{
${ }^{1} \mathrm{http}: / / \mathrm{www} . j o h n s t o n s a r c h i v e . n e t / a s t r o / a s t e r o i d m o o n s . h t m l \# 1$ is used for the list of asteroids. Relevant
} references and discovery dates of asteroids are given in the website (Last access: March 29, 2016). 
Table 1. The mean and median values of various properties of binary asteroids with known densities

\begin{tabular}{llll}
\hline \hline & Mean & Median & $\begin{array}{l}\text { Standard } \\
\text { Deviation }\end{array}$ \\
\hline Primary diameter $[\mathrm{km}]$ & 1.37 & 1.04 & 0.95 \\
\hline Secondary diameter $[\mathrm{km}]$ & 0.33 & 0.30 & 0.19 \\
\hline Secondary-to-primary ratio & 0.28 & 0.25 & 0.19 \\
\hline Density $\left[\mathrm{kg} / \mathrm{m}^{3}\right]$ & 1717.6 & 1700 & 309.6 \\
\hline Semi-major axis $[\mathrm{km}]$ & 2.83 & 2.62 & 1.72 \\
\hline $\begin{array}{l}\text { Semi-major axis-to- } \\
\text { primary ratio }\end{array}$ & 2.20 & 1.95 & 0.86 \\
\hline \hline
\end{tabular}

Our representative hypothetical binary asteroid, hereafter named as $2016 \mathrm{JGCD}$, is summarized in Table 2 . This assumes that both primary and secondary have a spherical shape and same constant density. According to the generally accepted binary formation hypothesis, binary primaries are like rubble-pile structures, and secondaries are originated from them. As a consequence, large differences in density are not expected between the two, thus the same density is assumed for both bodies. Finally, a perfectly circular orbit is used to model the motion of the secondary, and its period can be straightforwardly computed using the period of a Keplerian orbit.

In order to define a sensible representation of a typical binary asteroid, median values are used to define the main features of our notional binary $2016 \mathrm{JGCD}$, instead of using mean values, which could pose issues with both the skewness of the distribution and biased extreme values. The hypothetical binary asteroid will thus have $1000 \mathrm{~m}$ of primary diameter, $250 \mathrm{~m}$ of secondary diameter, $1950 \mathrm{~m}$ mutual orbit semi-major axis, and density of $1700 \mathrm{~kg} / \mathrm{m}^{3}$. However, as will be discussed later, the results of this paper can be easily scaled to any primary size, as long as the primary-secondary diameter ratio and the semi-major axis-primary radius ratio remain constant.

Table 2. The properties of hypothetical binary asteroid 2016 JGCD

\begin{tabular}{lll}
\hline \hline & Primary & Secondary \\
\hline Diameter $[\mathrm{m}]$ & 1000 & 250 \\
\hline Density $\left[\mathrm{kg} / \mathrm{m}^{3}\right]$ & \multicolumn{2}{c}{1700} \\
\hline Mass $[\mathrm{kg}]$ & $8.9 \times 10^{11}$ & $1.4 \times 10^{10}$ \\
\hline $\begin{array}{l}\text { Mutual orbit semi- } \\
\text { major axis }[\mathrm{m}]\end{array}$ & & 1950 \\
\hline
\end{tabular}




\begin{tabular}{l}
\hline \hline Orbital period $[\mathrm{h}]$ \\
\hline \hline
\end{tabular}

\section{Dynamical Framework}

This paper seeks unpowered/ballistic landing opportunities for small spacecraft, or science packages. Such a definition therefore is relevant to spacecraft, whose design allow no, or very limited, means of orbit and landing control, due to constraints in complexity, mass and/or volume. Hence, the work presented is applicable to small legged landers with only attitude control or, potentially, to NanoSat/CubeSat opportunity payloads. In either case, it is envisaged that the lander is deployed from a mothership in a safe trajectory near the binary system. Once deployed, the lander could follow the natural paths connecting regions far from the barycentre of the system, where the mothership would be stationed, with the surface of the asteroids.

These natural paths are sought within the framework provided by the Circular Restricted Three Body Problem (CR3BP). In CR3BP, two bodies are assumed to rotate around their common centre of mass, in circular orbits, under the point mass gravitational attraction of each other. The motion of the third body, in this case the lander, is determined by the gravitational interaction with the two large bodies in the system. The mass of the third body however is assumed to be sufficiently small so that its gravitational interaction with the other objects in the system can be neglected.

In the following subsections, the equation of motions of the CR3BP and its integral of motion are described.

\section{A. Equations of Motion}

The CR3BP is described in a rotating reference frame, located in the barycentre of two main bodies, whose $\hat{\mathbf{x}}$ axis points the secondary body, $\hat{\mathbf{z}}$ axis normal to the mutual orbit plane and $\hat{\mathbf{y}}$ axis completing the triad.

The only force acting on the third body (in this case, spacecraft) is the gravitational attraction from the other two bodies (primary and secondary asteroids). The motion of the spacecraft is then represented by: 


$$
\begin{aligned}
& \ddot{r}_{x}-2 \dot{r}_{y} \omega-r_{x} \omega^{2}+\frac{\mu_{1}\left(r_{x}+r_{10}\right)}{l_{1}^{3}}+\frac{\mu_{2}\left(r_{x}-r_{20}\right)}{l_{2}^{3}}=0 \\
& \ddot{r}_{y}+2 \dot{r}_{x} \omega-r_{y} \omega^{2}+\left(\frac{\mu_{1}}{l_{1}^{3}}+\frac{\mu_{2}}{l_{2}^{3}}\right) r_{y}=0 \\
& \ddot{r}_{z}+\left(\frac{\mu_{1}}{l_{1}^{3}}+\frac{\mu_{2}}{l_{2}^{3}}\right) r_{z}=0
\end{aligned}
$$

where $\omega$ denotes the angular speed of the mutual orbit, $\mu_{\mathrm{i}}(i=1,2)$ denotes the gravitational constant of primary and secondary bodies, $l_{i}$ denotes the distances from the primary and the secondary to the third body and $r_{i 0}$ denotes the distance from barycentre to the primary and the secondary.

Eq.(1) can be rewritten with the following normalizations: All the distances are normalized by the distance between primary and secondary, denoted by $r_{12}$, and the unit mass becomes the sum of the masses of the two bodies. The system mass parameter $\mu$ is defined as:

$$
\mu=\frac{m_{2}}{m_{1}+m_{2}}
$$

Finally, time is normalized with the angular speed of the synodic frame $\omega$, and as a result the universal gravitational constant also becomes unity.

Note that in this paper primary and secondary asteroids are assumed to have a spherical shape and equal density. Hence, in this case, the system mass parameter $\mu$ can be rewritten in a more convenient form as:

$$
\mu=\frac{n^{3}}{n^{3}+1}
$$

where $n$ is the secondary-to-primary ratio. For the hypothetical binary asteroid described in Table 2 , $\mu$ becomes 0.0154 .

If the normalized position vector is described as $\vec{r}=\left[\begin{array}{lll}x & y & z\end{array}\right]^{T}$, Eq. (1) becomes: 


$$
\begin{gathered}
\ddot{x}-2 \dot{y}=\frac{\partial U}{\partial x}=x-\frac{(1-\mu)(x+\mu)}{\rho_{1}^{3}}-\frac{\mu(x-1+\mu)}{\rho_{2}^{3}} \\
\ddot{y}+2 \dot{x}=\frac{\partial U}{\partial y}=y-\frac{(1-\mu) y}{\rho_{1}^{3}}-\frac{\mu y}{\rho_{2}^{3}}=y\left(1-\frac{1-\mu}{\rho_{1}^{3}}-\frac{\mu}{\rho_{2}^{3}}\right) \\
\ddot{z}=\frac{\partial U}{\partial z}=-\frac{(1-\mu) z}{\rho_{1}^{3}}-\frac{\mu z}{\rho_{2}^{3}}=z\left(-\frac{1-\mu}{\rho_{1}^{3}}-\frac{\mu}{\rho_{2}^{3}}\right)
\end{gathered}
$$

where $U$ is the potential function, which is the sum of gravitational and centrifugal potential:

$$
U(x, y, z)=\frac{1}{2}\left(x^{2}+y^{2}\right)+\frac{1-\mu}{\rho_{1}}+\frac{\mu}{\rho_{2}}
$$

Note that because of the adimensionality of Eq.(4) the results computed here are actually applicable to any binary with density, secondary-to-primary and semi-major axis to primary ratios as reported in the median results of Table 1 . Hence, the results presented are relevant to a whole range of binary sizes. However, the paper focuses on the notional 2016 JGCD binary, and thus, for clarity, the results are scaled to the units corresponding to the 2016 JGCD case.

\section{B. Jacobi Constant}

The mechanical energy in the CR3BP is represented by the Jacobi Constant $C$. It is a non-dimensional constant, which could be obtained by solving the Jacobi Integral in the rotating reference frame, and described as below [20]:

$$
C=2 U-V^{2}
$$

where $V$ is the spacecraft speed given in rotating frame of reference. Note that since the Jacobi Constant corresponds to $C=-2 E$, where $E$ is classical mechanical energy (i.e. $V^{2} / 2-\mathrm{U}$ ), decreasing energy is equivalent to increasing $C$. Bearing this in mind, the terms energy and Jacobi Constant are used interchangeably throughout the text.

For a given set of initial conditions, Jacobi Integral defines the accessible and forbidden zones of motion. From the expression in Eq.(6), it can be deduced that there are locations, for which at some energies, the velocity of the motion may become an imaginary number, which clearly indicates the physical impossibility of motion within those locations, at those energy levels. Hence, zero-velocity surfaces (ZVS) are defined when one considers the condition $V^{2}=0$. ZVS shows which regions are accessible to motion at a given energy.

The Jacobi Constant can then define five clearly distinguishable regimes of motion: For high values of Jacobi Constant, ZVS show two spheres (bubbles) around the two large bodies, as well as an exterior region (see Fig. 2). 
The motion is thus restricted either to the vicinity of primary or secondary, or to the exterior region (i.e., no connections between these three bubbles exist). Decreasing the Jacobi Constant, which means increasing the energy, gradually connects these two spheres and eventually makes the motion initially possible only between the two bodies, and later with exterior region too. Finally, when Jacobi Constant is sufficiently small, all 3D space becomes accessible (i.e. $\mathrm{C} \leq 0)$.

There are critical points at which the bubbles around each body and the exterior region connect. These critical points correspond to energies of the collinear Lagrange points along the x-axis in rotating frame. The first connection occurs between the inner regions where L1 point is present. It is followed by L2 point which connects inner region to exterior region, then another connection to exterior region occurs at L3 point. The final regions of unavailable motion near the orbital plane of the secondary are those near the L4 and L5 equilibrium points, which disappear simultaneously for Jacobi Constants smaller than that of the equilateral equilibrium positions.

As the L2 is the very first point that connects inner region to exterior region, it provides the lowest energy gate to enter into the proximity of the asteroid surface.

\section{Design of Landing Trajectories}

The mission architecture considered in this paper is that of a mothership which carries one (or several) landers. Once the mothership reaches the binary system, it would start orbiting near the binary and perform the necessary science operations. The mothership should be sufficiently close to the binary system to perform satisfactorily detailed observations; however, it should also remain sufficiently far away from the system's barycentre to prevent contamination of instruments and sensors, as well as the spacecraft itself, due to the unfavourable asteroid environment. Such an operational orbit would most likely be in the exterior region of zero-velocity surfaces closed at $\mathrm{L} 2$ point. The operational orbit would also prevent the spacecraft to collide with the binary system, as there is no motion possible to the interior region. Fig. 2 shows a general illustration of proposed mission architecture. 


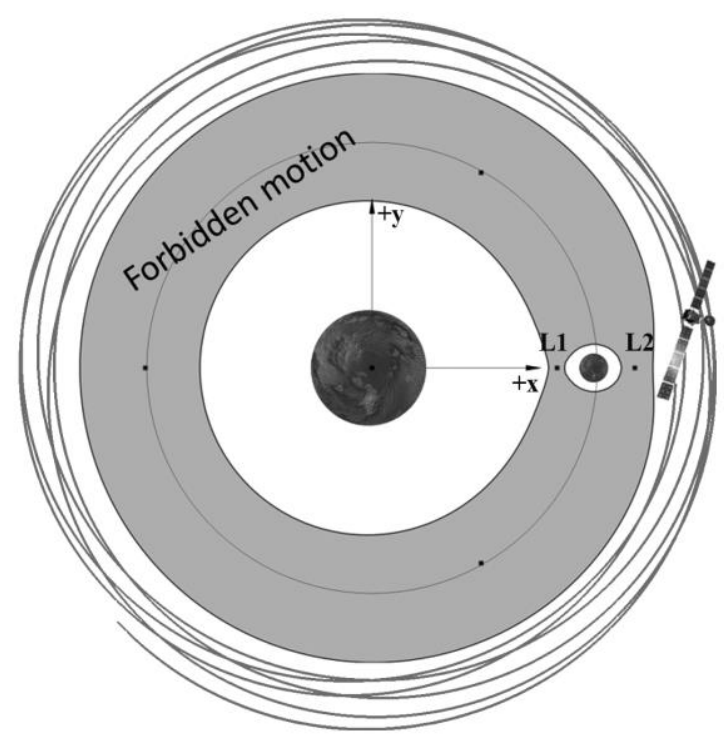

Fig. 2 Illustration of mission architecture

The lander deployment shall then be performed while the mothership is in its operational orbit in the exterior region, as depicted in Fig. 2. It is envisaged that the mothership is equipped with a simple spring mechanism that would be used to deploy the lander. The spring mechanism is thus used to provide sufficient speed to open the ZVS at the $\mathrm{L} 2$ point. The Philae lander, for example, was deployed with an adjustable push-off device allowing a range of deployment speeds between 0.05 to $0.52 \mathrm{~m} / \mathrm{s}$ [21]. Rosetta also carried an emergency spring release system designed to eject Philae at $0.17 \mathrm{~m} / \mathrm{s}$ [21]. On the other hand, current standardized deployers for CubeSats, such as the Poly Picosatellite Orbital Deployer (PPOD) [22], eject the CubeSat payloads at $2 \mathrm{~m} / \mathrm{s}$, approximately.

Landing trajectories are thus sought that allow this hypothetical unpowered spacecraft to land on a predetermined site on the surface of one of the asteroids in the system. A key requirement for these landing opportunities is to allow for the slowest possible touchdown speed; firstly, to prevent damage to the spacecraft and its payload and, secondly, to minimize the risk of bouncing off the system if sufficient damping of the energy at touchdown is not achieved. This paper considers a simple model for the interaction of the spacecraft with the asteroid's surface; that of a bouncing ball with a specified coefficient of restitution $\varepsilon$ [23].

Hence, given the initial conditions of the spacecraft after deployment, Eq. (4) can be forward integrated up to the point on which the trajectory intersects the surface of one of the asteroids in the system. The velocity of the corresponding state vector defines the touchdown conditions, and can be described in a local-vertical (LV), localhorizontal (LH) components as: 


$$
\begin{aligned}
\mathbf{v}_{L V}^{-} & =(\hat{\mathbf{n}} \cdot \mathbf{v}) \hat{\mathbf{n}} \\
\mathbf{v}_{L H}^{-} & =\mathbf{v}-(\hat{\mathbf{n}} \cdot \mathbf{v}) \hat{\mathbf{n}}
\end{aligned}
$$

where the vector $\hat{\mathbf{n}}$ corresponds to the normal to the surface at the contact point, and the superscript (-) refers to the conditions just an infinitesimal instant before the rebound.

Considering an impulsive rebound at touchdown with some loss of energy due to, for example, the deformation of the surface and/or spacecraft, the velocity conditions an infinitesimal instant after the rebound can be at first approximation modelled as:

$$
\begin{aligned}
& \mathbf{v}_{L V}^{+}=-\varepsilon_{V}(\hat{\mathbf{n}} \cdot \mathbf{v}) \hat{\mathbf{n}} \\
& \mathbf{v}_{L H}^{+}=\varepsilon_{H} \mathbf{v}_{L H}^{-}
\end{aligned}
$$

where the coefficient of restitution $\varepsilon_{V}$ defines the dissipation of energy in local vertical direction, while $\varepsilon_{H}$ defines that of the local horizontal, or tangential, direction. These coefficient must typically have a value between 0 and 1 , although, as shown in Tardivel et al. [24] and Biele et al. [25], the values of $\varepsilon_{V}$ and $\varepsilon_{H}$ may differ substantially, since the interaction of the vertical and tangential motion with the surface are fundamentally different. Note also that the model in Eq.(8) assumes that the outgoing velocity will remain in the plane defined by the incoming velocity and vector $\hat{\mathbf{n}}$. Irregularities in the surface, such as boulders or sharp surface features, may change this, however, this possibility is not considered in the paper.

\section{A. Landing and Deployment}

Hence, we seek to find deployment state vectors $\left(x_{d}, y_{d}, z_{d}, \dot{x}_{d}, \dot{y}_{d}, \dot{z}_{d}\right)$ that satisfy the landing conditions described above. These are six completely unknown design variables. However, if the landing site is defined beforehand by its synodic latitude $\phi$ and longitude $\lambda$, and recalling that both primary and secondary objects are assumed to be spherical and landing trajectories are sought with only local vertical touchdown velocity, the landing state vector in a body-centred body-fixed frame (BCBF) would be of the form:

$$
\left\{\begin{array}{l}
x=r_{i} \cos \lambda \cos \phi \\
y=r_{i} \sin \lambda \cos \phi \\
z=r_{i} \sin \phi \\
\dot{x}=-v_{l} \cos \lambda \cos \phi \\
\dot{y}=-v_{l} \sin \lambda \cos \phi \\
\dot{z}=-v_{l} \sin \phi
\end{array}\right.
$$


where the subscript $i$ refers to the primary if $i=1$ or to the secondary if $i=2$ and $v_{1}$ is the magnitude of the touchdown or landing speed. Note that, as discussed earlier, most of the secondaries are tidally locked, hence, their attitude can be assumed fixed in the synodic reference frame as well, and the body's prime meridian $(\lambda=0)$ can be arbitrarily defined as on the $x$-axis and on the far side of the system (facing the classical L2 point as in Fig. 2). Hence, the landing state vector on the secondary can be described by Eq. (9) adding only the position of its barycentre, i.e. (1$\mu, 0,0)$.

However, the primary will have a fast rotation, which is likely to be near the point at which a body with negligible cohesion starts shedding mass [14]. As a standard value and configuration, we assume here that the rotation of the primary is $2.2 \mathrm{~h}$, and its rotational axis is also perpendicular to the orbital plane of the secondary [14, 18]. Hence the state vector of landing conditions on the primary can be represented as:

$$
\left\{\begin{array}{l}
x=r_{1} \cos \lambda \cos \phi-\mu \\
y=r_{1} \sin \lambda \cos \phi \\
z=r_{1} \sin \phi \\
\dot{x}=-\left(v_{l} \cos \lambda+\left(\omega_{1}-\omega\right) r_{1} \sin \lambda\right) \cos \phi \\
\dot{y}=-\left(v_{l} \sin \lambda-\left(\omega_{1}-\omega\right) r_{1} \cos \lambda\right) \cos \phi \\
\dot{z}=-v_{l} \sin \phi
\end{array}\right.
$$

where $\omega_{1}$ is the normalized angular rotation of the primary, so that $\omega=1$. Note finally that for the primary, the longitude $\lambda$ in Eq. (10) only provides information of the landing longitude in the synodic reference frame, instead for the secondary, since this is tidally locked, this longitude also represents the actual meridian on the BCBF frame.

Hence, given the landing state vector as defined by the synodic latitude $\phi$ and longitude $\lambda$ pair of its landing site, and its touchdown speed $v_{1}$, the trajectory can be propagated backwards until some satisfactory deployment condition is found. Note that once the landing site is defined, only one design parameter requires to be determined, this is the touchdown speed $v_{1}$.

A bisection algorithm search, inspired by Ren \& Shan [26], is then implemented to determine the minimum possible touchdown speed $v_{1}$ for a given landing site.

As with any bisection method, firstly a lower and an upper threshold for $v_{1}$ need to be determined. The lower threshold must be such that the resultant trajectory would never reach the exterior region of the system (no-escape condition). On the other hand, an upper threshold for $v_{1}$ must generate a landing trajectory such that reaches the 
exterior region of the system and so deployment opportunities occur (escape condition). At each iteration $i$ of the algorithm, the landing trajectory corresponding to a speed $v_{l}^{i}$ such as:

$$
v_{l}^{i}=\frac{v_{l}^{U B}+v_{l}^{L B}}{2}
$$

is propagated backwards. Here, $v_{l}^{L B}$ is the lower bound and $v_{l}^{U B}$ is the upper bound speed.

A trajectory is said to be a feasible landing trajectory if it reaches the exterior region of the binary system. This exterior region is defined as the volume of space, outside the orbit of the secondary, where the motion is allowed with the Jacobi constant of the $\mathrm{L} 2$ equilibrium point $C_{L 2}$. Trajectories that narrowly miss the surface of one of the asteroids $\left(<1.25 r_{\mathrm{i}}\right)$, except during the final descent, or that spend less than $8 \mathrm{~h}$ in the exterior region, are also considered unfeasible landing conditions. These final constraints into the trajectories, although relatively ad-hoc, are added to ensure that the window for a deployment is sufficiently long and that the trajectory does not require more than one close approach to the object before landing. The latter is an attempt to avoid landing conditions that would be extraordinarily sensitive to the intrinsic epistemic uncertainties of the asteroid gravitational potential.

The algorithm iterates the above procedure and substitutes the attempted speed $v_{l}^{i}$ for the upper boundary $v_{l}^{U B}$, if $v_{l}^{i}$ lead to an escape trajectory, or for the lower boundary $v_{l}^{L B}$, if it did not. The algorithm stops once a certain tolerance is reached.

The initial lower and upper bounds of the bisection algorithm are defined based on the no-escape/escape conditions that were previously mentioned. Clearly, no-escape condition would occur for $v_{1}=0$, but a larger lower threshold can also be identified if the energy of the L2 point is used to compute the $v_{1}$, since at this energy there is no possible escape condition. On the other hand, obviously, for escape condition to happen for $v_{l}^{U B}$ the state vector in the synodic reference frame must be such that ZVS are opened at the L2 Point.

A good first guess approximation of the minimum touchdown speed as a function of landing site can thus be computed as:

$$
\begin{aligned}
& v_{l 1}^{L B}=\sqrt{\left(x^{2}+y^{2}+2 \frac{1-\mu}{\rho_{1}}+2 \frac{\mu}{\rho_{2}}-C_{L 2}-\left(\omega_{1}-\omega\right)^{2} r_{1}^{2}\right)\left(1+\sin \phi^{2}\right)^{-1}} \\
& v_{l 2}^{L B}=\sqrt{x^{2}+y^{2}+2 \frac{1-\mu}{\rho_{1}}+2 \frac{\mu}{\rho_{2}}-C_{L 2}}
\end{aligned}
$$


where $v_{l 1}^{L B}$ and $v_{l 2}^{L B}$ are the minimum touchdown speeds in the primary's and secondary's BCBF frame and $\rho_{1}$ and $\rho_{2}$ are the distance to the primary and secondary objects, respectively. Eq.(12) are derived by estimating the Jacobi constant of the L2 equilibrium point $C_{L 2}$, substituting it in Eq.(6) and solving for landing speed. Fig. 3 shows the resulting values of Eq.(12), with the dimensions corresponding to the hypothetical binary asteroid 2016 JGCD, as described in Table 2.
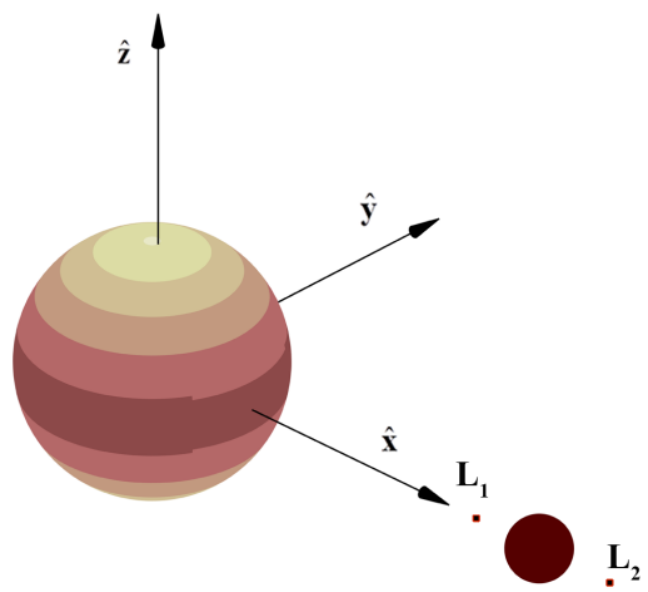
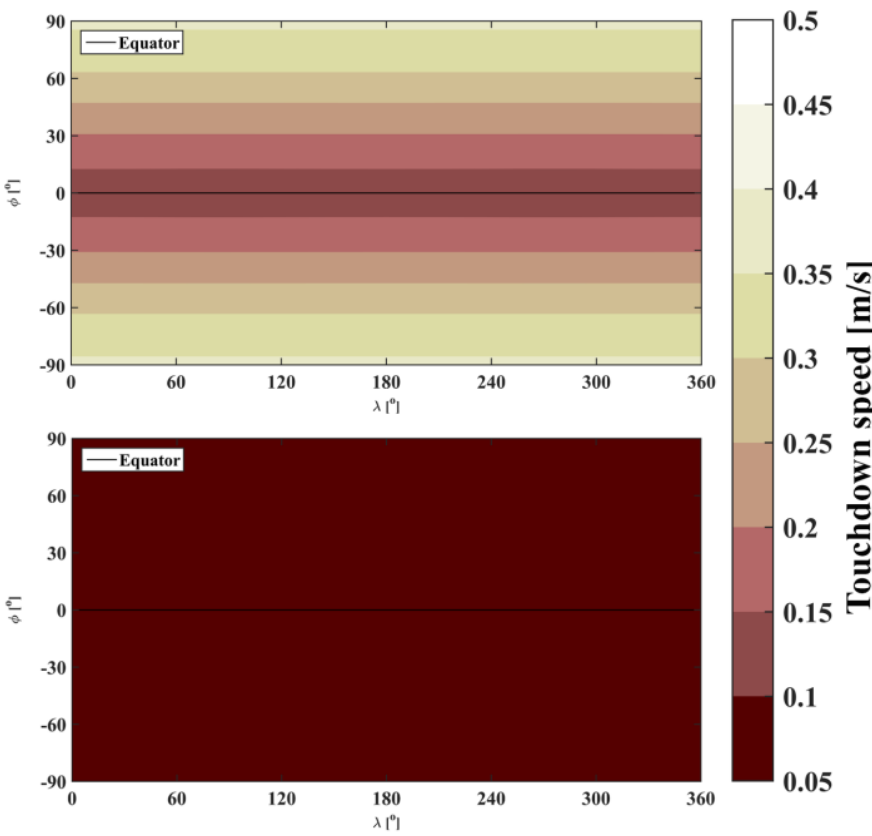

Fig. 3 Minimum touchdown speed as a function of landing $v_{l 1}^{L B}$ (above) and $v_{l 2}^{L B}$ (below), computed as in

Eq.(12). The minimum touchdown speed on the secondary is only fractionally varying over the surface and around $0.08 \mathrm{~m} / \mathrm{s}$. The variation over the primary surface is mainly caused by its rotation. Note that the classical (i.e. Keplerian) escape velocity from the surface of the primary would be $\sim 0.5 \mathrm{~m} / \mathrm{s}$.

The upper bound is instead simply defined by multiplying the lower boundary with some arbitrary, relatively large number.

Once the resulting trajectories are obtained and propagated backwards for a sufficiently long timespan, the exact deployment location of the landing trajectory can be chosen within the propagated path. In principle, any point beyond the L2 point can be seen as a deployment location. Recall that the mothership is assumed to be orbiting the binary system in an operational orbit such that the ZVS are closed at the L2 point (i.e., exterior realm or region). The 
deployment speed can then be thought as a manoeuvre to provide the lander spacecraft with sufficient speed to open up the ZVS and enable the landing path computed beforehand [27].

Hence, let the mothership have an orbital speed such as $v_{\text {mother }}(\mathbf{r})$, which for each feasible point of deployment in the landing path must be such that:

$$
C_{L 2} \leq 2 U(\mathbf{r})-\left(v_{\text {mother }}(\mathbf{r})\right)^{2}
$$

where $\mathbf{r}$ denotes a position vector in the landing path where the deployment could be implemented. Hence, the minimum deployment speed $\Delta v$ in a position $\mathbf{r}$ must then satisfy the following equality constraint:

$$
C_{L 2}=2 U(\mathbf{r})-\left(v_{\text {lander }}(\mathbf{r})+\Delta v(\mathbf{r})\right)^{2}
$$

where $v_{\text {lander }}(\mathbf{r})$ is the speed of the lander at the position $\mathbf{r}$ in the landing path, as computed by the backward propagation. Finally, the deployment speed $\Delta v$ can be estimated by solving Eq.(14) for $\Delta v$ :

$$
\Delta v(\mathbf{r})=\sqrt{2 U(\mathbf{r})-C_{L 2}}-v_{\text {lander }}(\mathbf{r})
$$

Note that the $\Delta v$ obtained as above is only the minimum manoeuvre to reach the $\mathrm{L} 2$ point energy, hence assumes that the mothership's operational orbit has a Jacobi Constant of exactly $C_{L 2}$. However, the mothership is likely to be in more stable configurations, with higher Jacobi constants. The latter discussion, as well as the exact direction of the manoeuvre, which will depend on the actual mothership's operational orbit is not explored in this current paper.

Finally, it is noteworthy to mention that since spherical asteroids are being used, as well as due to the symmetry of motion around the reference plane of the CR3BP, landing trajectories for the northern hemisphere are the mirror image of those in the southern hemisphere. This means that the direction of landing conditions of mirror image latitudes will differs on the surface, however the magnitude will not. Nevertheless, the same does not occur with an $\mathrm{X}-\mathrm{Z}$ partition, since there is not symmetry of motion due to the rotation of the reference frame.

\section{B. Primary landing results}

Regardless of whether the landing occurs on the primary or on the secondary, all previous discussion on minimum energy landing still holds. Thus, landings on the primary are also sought by assuming the lowest possible energy, which is that of the opening of the L2 bottleneck. However, the results show that the required energies to land on the primary are much higher than that. As shown in Fig. 4, even the equatorial region, which is the most accessible region of the primary, requires Jacobi Constants $\mathrm{C}<<\mathrm{C}(\mathrm{L} 4 / \mathrm{L} 5)$. At these energies, ZVS are nearly all 
open. Only some out-of-plane regions remain unavailable for motion until $\mathrm{C}<0$, hence the higher energies at the poles. In terms of the actual touchdown speeds, the equator allows ballistic landing with touchdown velocities slightly below $30 \mathrm{~cm} / \mathrm{s}$. Note that this is nearly twice as much as the estimate for the minimum touchdown speeds $v_{l 1}^{L B}$ as computed by Eq.(12). The touchdown speed increases with increasing latitude, reaching $45 \mathrm{~cm} / \mathrm{s}$ at the poles, which is a similar estimate to that of Eq.(12). Note that the two body escape velocity for the notional 2016 JGCD at the surface of the primary is computed to be $\sim 50 \mathrm{~cm} / \mathrm{sec}$.

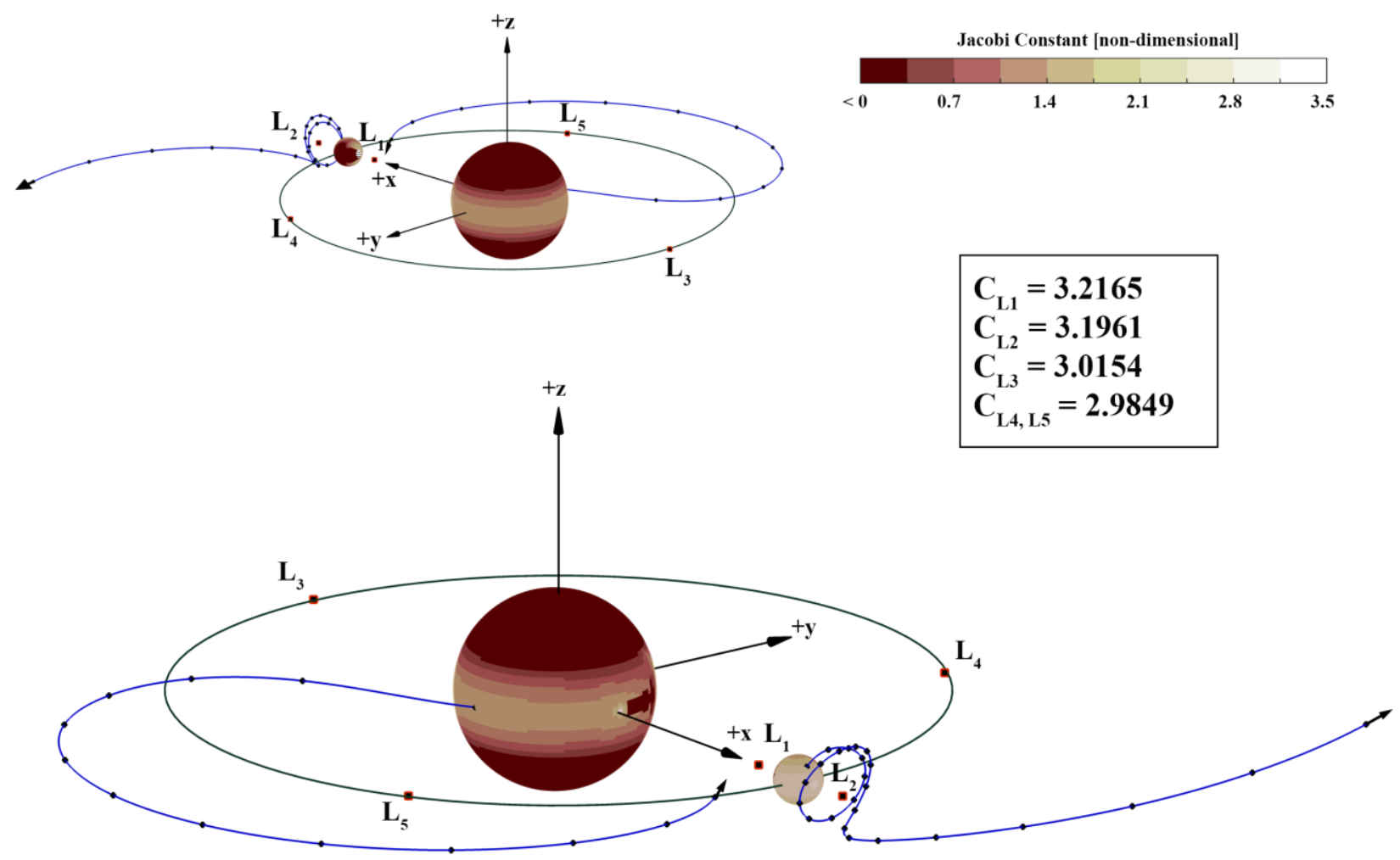

Fig. 4 Energy map of trajectories on both primary and secondary. C values in the box show the Jacobi Constants of each Lagrange point. Dots in the trajectories denote $1 \mathrm{~h}$ motion marks.

Fig. 4 shows two example trajectories that land on the primary and secondary, as well as the asteroid surfaces coloured with the level of Jacobi Constant of the corresponding landing trajectory. The reader should note the maximum Jacobi Constant value used in the colour bar: Trajectories requiring Jacobi Constants $\mathrm{C}<0$ are all coloured at the same level. 


\section{Secondary landing results.}

Unlike the relative straightforwardness of the landing trajectories on the primary, landing on the secondary shows much richer dynamics. In general, much lower energy landing opportunities than in the primary can be observed as depicted with Fig. 4 colour map. Fig. 5 provides more detail to the resultant Jacobi Constant for landings onto the secondary.

As observed in Fig. 5, the Jacobi Constant of trajectories landing on the secondary is intensely affected by the secondary's rotation around the primary. In general, the regions that are facing the L2 points are reachable by the lowest energy trajectories. However, trajectories landing in the same direction of the tangential motion of the secondary are also characterised by lower energies. Fig. 5 shows the two hemispheres of secondary surface as cuts in X-Z plane. The eastern hemisphere is seen in the left hand side of Fig. 5, from the longitude $0^{\circ}$ to $180^{\circ}$. The letter "N" shows the $+\mathrm{Z}$ direction, i.e. $90^{\circ}$ latitude. The western hemisphere can be seen on the right hand side, from the longitude $180^{\circ}$ to $360^{\circ}$ (i.e. equal to $0^{\circ}$ ). The starting point (i.e. landing site) of the example trajectory is also marked with an arrow in the direction of the backward propagation (same example trajectory as in Fig. 4). The cross-like symbol indicates that $+\mathrm{Y}$ is out of the page direction. The dot symbol on the right hand side indicates that $+\mathrm{Y}$ direction is into the page. The Jacobi Constant of the landing trajectories onto the different landing sites can be observed in the figure.

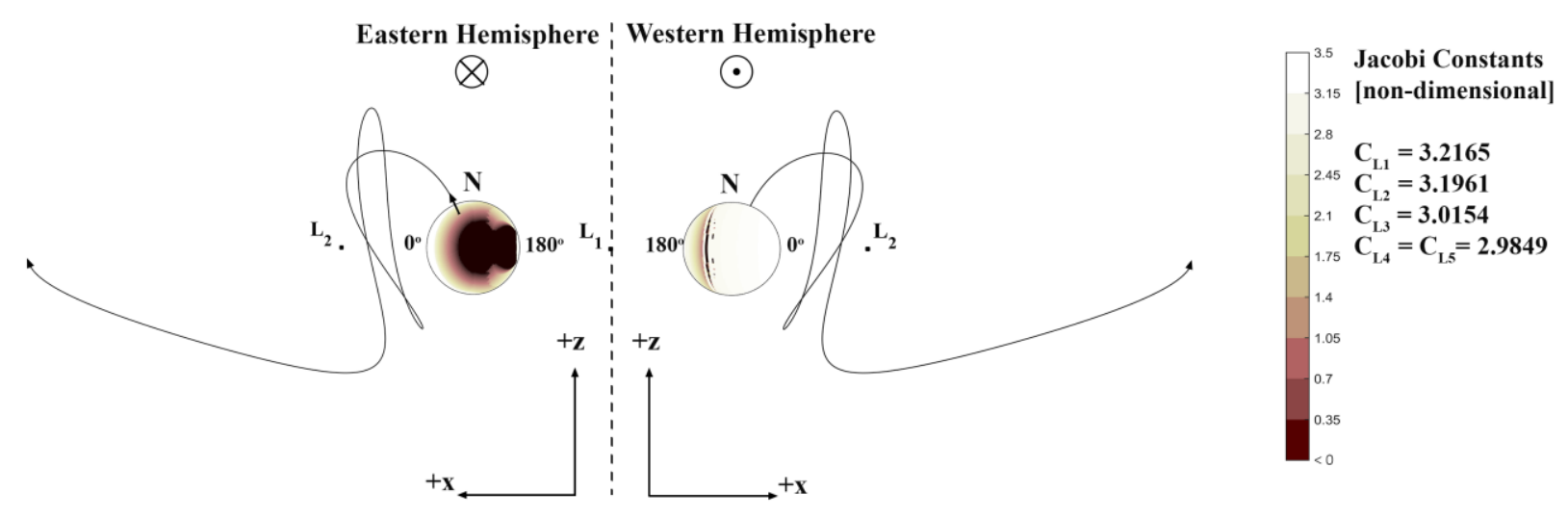

Fig. 5 Energy map of trajectories to land on secondary surface (C values show the Jacobi Constants of Lagrange points.)

The rotation of the binary system appears to be affecting the energy of the landing trajectories. If the landing is performed on a site in the eastern hemisphere of the secondary, the motion of the secondary, in the opposite 
direction of the landing, seems to be energizing the trajectories. On the other hand, landing in the western hemisphere requires less energy. In this latter case, the lander is catching a secondary that moves on the same direction. A third of the equatorial region is observed to be reachable without increasing the energy further than that to open up L3. This ratio decreases at higher latitudes.

The lowest energy trajectories, whose energies are indicated with white colour in Fig. 5, generally perform a partial or complete revolution/orbit around L2 point before clearing off to the exterior region: Hence, showing a clear association with periodic orbits near the L2 point, i.e., transit trajectories through a narrow L2 point bottleneck. Accordingly, it also takes longer to land for such trajectories, as can be observed in the example in Fig. 5. This issue will be further discussed in the following section. Higher energy trajectories (grey and black coloured regions) are instead more direct trajectories, taking less time to land.

There is also a varied taxonomy of landing trajectories with different topologies at energies near that of the equilateral equilibrium positions L4/L5. At these energies only a narrow band of surface is available, extending from $200^{\circ}$ to $250^{\circ}$ longitude approximately. Among the mentioned varied topology of trajectories, some trajectories seem to perform different types of co-orbital motion for some significant periods of time. An example of this motion is reproduced in the following sub-section. Finally, as clearly marked in Fig. 6, a small region facing the primary cannot be reached from the exterior region with the required landing conditions. This is due to the fact that a landing constrained as in previous sections would always intersect the primary.

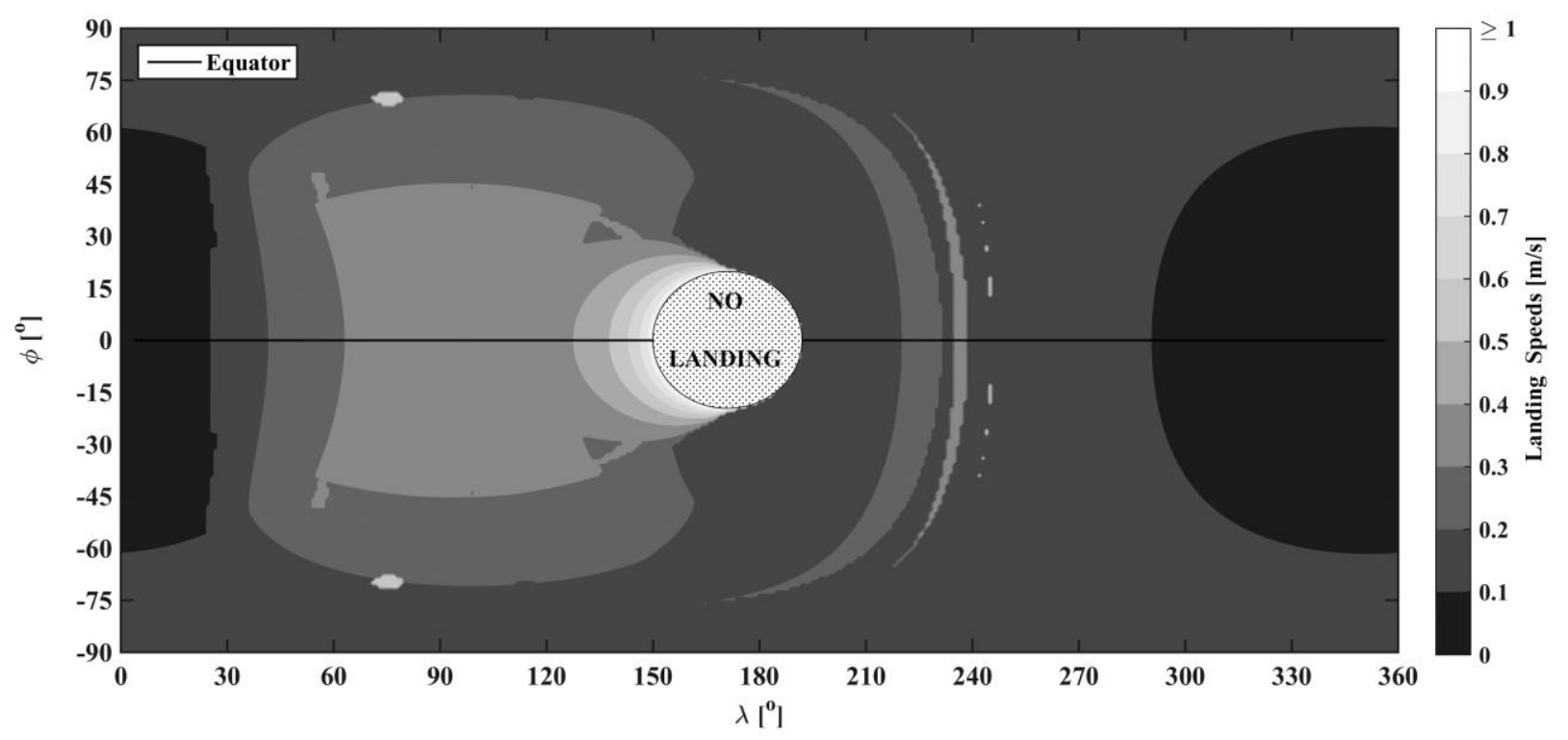

Fig. 6 Landing speed map over the surface of secondary. 
Fig. 6 summarises landing speeds on the surface of secondary. Note that the colormap is defined such that landing speeds larger than $1 \mathrm{~m} / \mathrm{s}$ are all coloured in white. This is done in order to increase the colour contrast for accessible regions with $<1 \mathrm{~m} / \mathrm{s}$ of touchdown. Clearly, the lower the energy of the trajectory is, the lower the landing speed becomes. Therefore, main features of Fig. 5 and Fig. 6 are very similar. Landing speeds are observed at magnitudes lower than $10 \mathrm{~cm} / \mathrm{s}$, with the minimum at $9.04 \mathrm{~cm} / \mathrm{s}$, as with dimensions corresponding to $2016 \mathrm{JGCD}$. On the other hand, the maximum speeds are observed in the regions where secondary faces the primary, with magnitudes near or above $1 \mathrm{~m} / \mathrm{s}$. It is also noteworthy that the majority of the secondary surface appears to be accessible with landing speeds lower than the corresponding two-body escape velocity. The latter is here computed as the sum of the two-body escape velocities for the primary and the secondary in the co-rotating frame, which at the furthest point from the barycenter (i.e. tip point of the secondary on the side facing the L2 point) is found to be approximately $35 \mathrm{~cm} / \mathrm{sec}$.

\section{Deployment results.}

As described earlier, deployment speeds can be computed at any position in the exterior realm, where the latter is defined as the region beyond the ZVS corresponding to the energy of the L2 point. Hence, in order to achieve the computed touchdown speeds for a given landing site, as shown in Fig. 6, this means that the results of deployment speed $\Delta v$, as in Eq.(15), are function of the synodic latitude $\phi$ and longitude $\lambda$ of the landing site and the time of

backward propagation. Note then that this time of backward propagation would correspond to the landing operation timespan or time from deployment to touchdown.

Since these results are four-dimensional, Fig. 7 summarises the deployment speeds for the equator only, hence synodic latitude $\phi=0^{\circ}$. X-axis shows the equatorial longitude, of which $0^{\circ}$ and $360^{\circ}$ longitude is the same point and facing the L2 point. Y-axis shows instead the landing operation timespan, i.e. time to reach the surface after the separation from the mothership, and different colours in the filled contour plot corresponds to deployment speeds. As an example, at $45^{\circ}$ longitude, for 6 hours landing duration, the mothership must provide about $0.1 \mathrm{~m} / \mathrm{s}$ at deployment. Also note the maximum deployment speed of the colour map; though the maximum deployment speed is determined to be $2 \mathrm{~m} / \mathrm{s}$, the colour map maximum is set to $0.5 \mathrm{~m} / \mathrm{sec}$ in order to improve the visualisation at lower deployment speeds. 


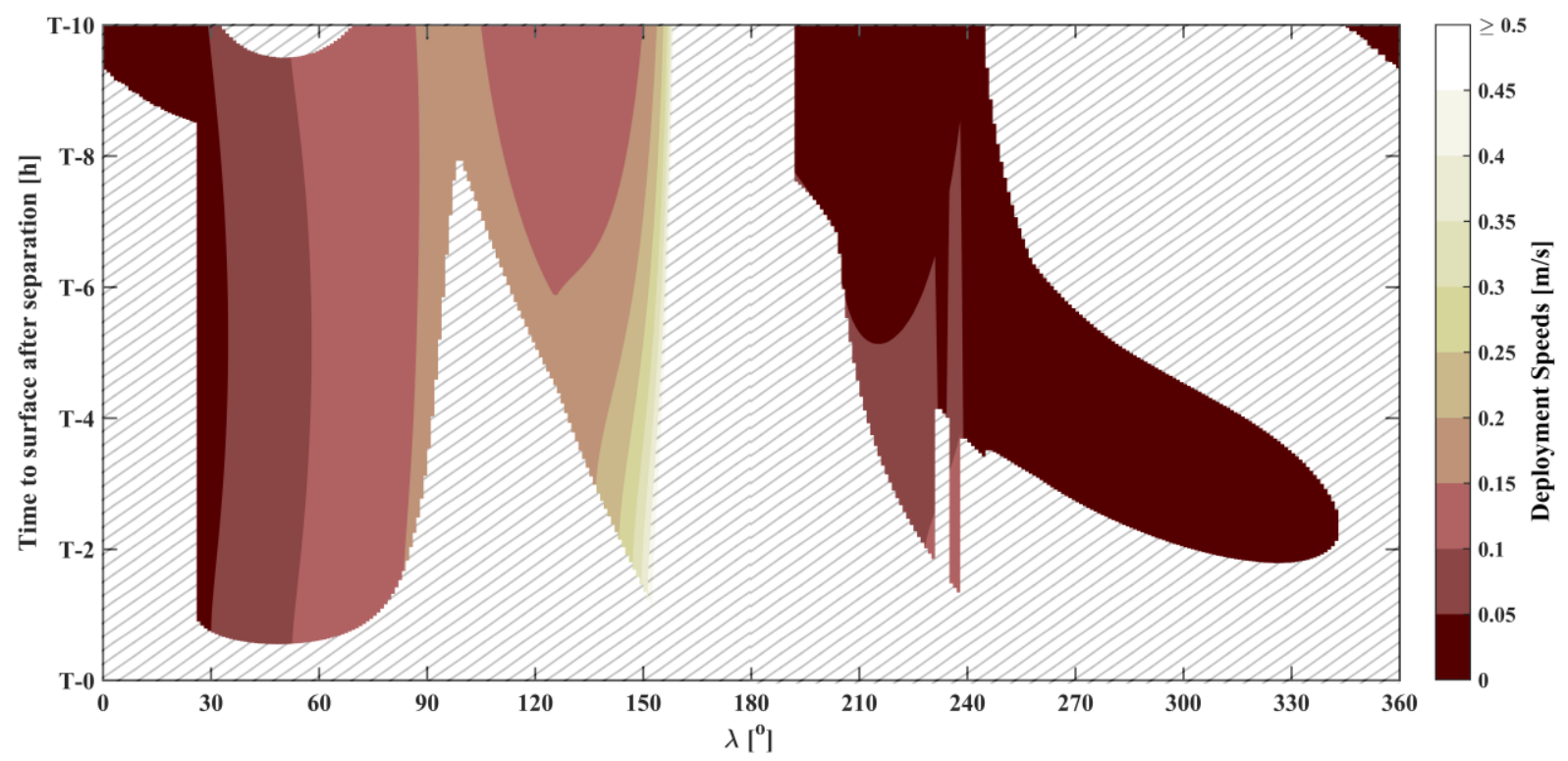

Fig. 7 Deployment speeds for landings in equatorial longitudes and landing durations 10 hours or less

For some of the lowest energy landing trajectories, i.e. mostly on the $\pm 30^{\circ}$ range from the prime meridian $(\lambda=0)$, the deployment location is required to be near the Lagrange L2 point, unless extremely long landing trajectories are considered. An example of such a trajectory can be seen in Fig. $8 a$, for an equatorial landing and longitude at $\lambda=0^{\circ}$. The landing trajectories shown in Fig. 8 were propagated backwards for four full synodic periods. Deployment options are shown only for the portions of trajectories that transit within the exterior realm (as defined earlier). Deployment within 10h of touchdown in Fig. $8 a$ corresponds only to the part of the trajectory performing a last loop around L2 point. Thus, the set of lowest energy landing conditions generally spends some time winding near the L2 point before definitively moving to the exterior region. During this motion around L2 point, some portion of the trajectory occurs just beyond the L2 distance, even before definitively moving out of this region, hence allowing for the first deployment options very near the Lagrange point. This is however a rather dynamically unstable location, therefore bringing mothership to this location and deploying a lander may entail important challenges.

Much more direct landing trajectories, with landing operations shorter than 4 hours, are also possible. However, these more direct options imply higher energies, often requiring the Jacobi Constant to be sufficiently low as to have the ZVS fully open. Some examples of these trajectories can be seen in Fig. $8 b$ to $8 c$. These higher energy options also require higher deployment speeds from the mothership. Fig. $8 c$ depicts a typical landing trajectory requiring landing speeds that are beyond the escape velocity of the system, hence the spiral shape trajectory appears as a 
consequence of the synodic reference frame (i.e. co-rotating with the secondary) used to represent the dynamics of the system.

Fig.8d-e depicts trajectories with energies near those of the triangular equilibrium positions L4 and L5. As mentioned earlier, these trajectories mostly occur within a narrow longitude band extending from $200^{\circ}$ to $250^{\circ}$, and depict a varied taxonomy of landing topologies. Fig8d shows a typical epicycle motion in the synodic reference frame. Fig8e instead shows a trajectory depicting co-orbital motion features, other trajectories show similar features but with complete loops around the L3 point (not shown in Fig 8). Finally, Fig $8 f$ depicts another trajectory with Jacoby Constant $0<\mathrm{C}<\mathrm{C}(\mathrm{L} 4 / \mathrm{L} 5)$. Recall that all simulation in Fig 8 were propagated for four full synodic periods, which in the notional 2016 JGCD asteroid would correspond to $\sim 3.2$ days.

In general, however, Fig. 7 depicts relatively low values for the required deployment speed for landing durations under 10 hours. The time necessary to reach the asteroid's surface however exhibits much more inhomogeneous features. For equatorial landing, separation speeds larger than $0.2 \mathrm{~m} / \mathrm{s}$ only occur in a relatively small range of longitudes between $148^{\circ}$ and $162^{\circ}$, near the boundary with the no-landing region. The $30^{\circ}$ wide region, spanning from $162^{\circ}$ to $192^{\circ}$ in longitude, depicts no deployment options. This region corresponds to the same no-landing region described in Fig. 6, where landing trajectories intersect the primary surface, before reaching the secondary. It is noteworthy to highlight again that the regions, where no landing trajectories are possible, are artificially affected as a consequence of the constrained landing geometry. It is very likely that allowing some additional horizontal velocity at touchdown, landing in those regions may also be possible, but this is simply not computed here. The paper is concerned with the overall insights of the minimum energy vertical landing in a binary system with predefined mission architecture and constraints, but it is clear that attempting to land on no-landing regions imply additional challenges due to the strong interaction of the landing trajectories with the other body in the system. In particular, landing trajectories that target the primary-facing side of the secondary pass extremely close to the primary surface, therefore uneven shape and irregular gravitational field of the primary are likely to perturb the trajectory. 

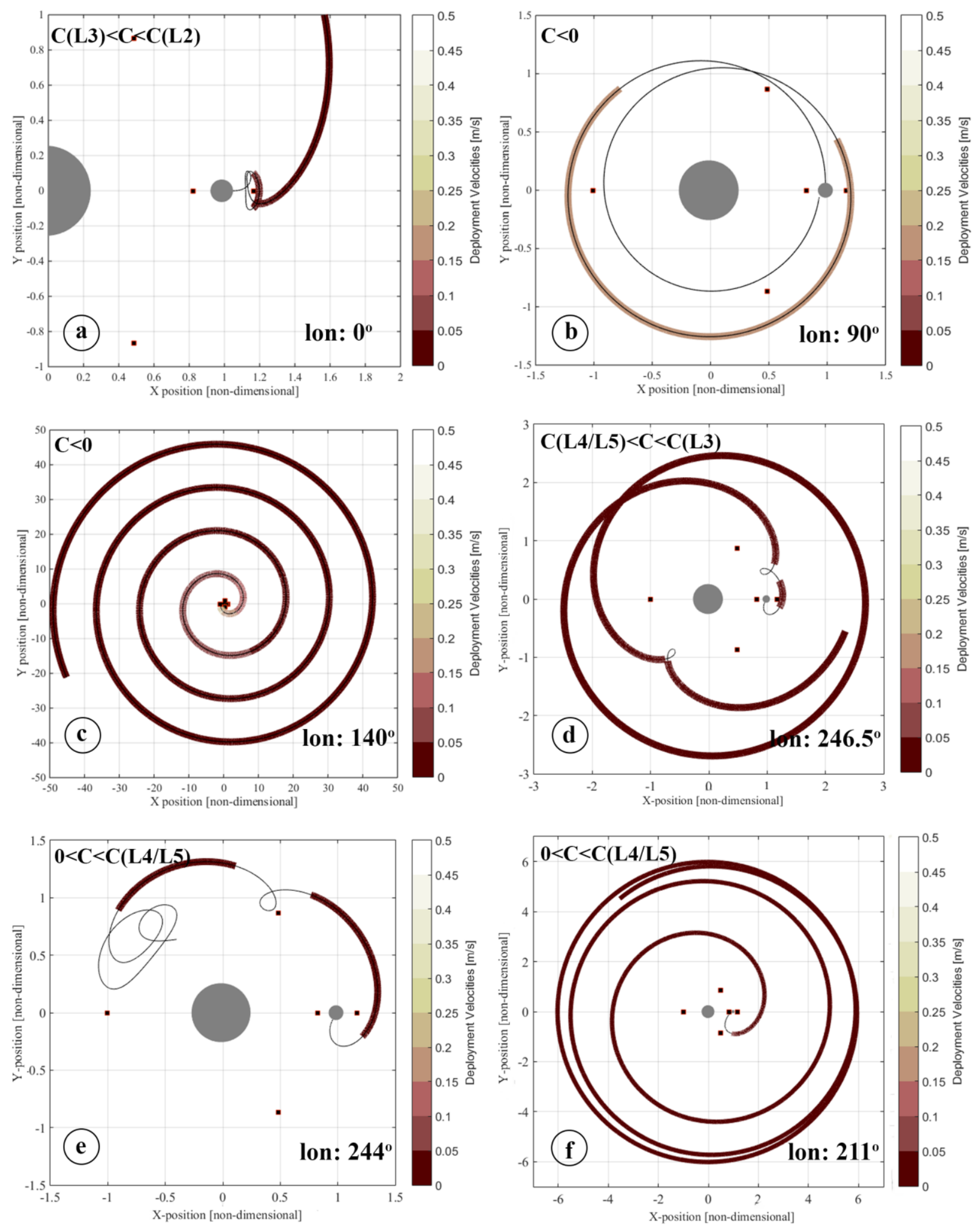
Fig. 8 Trajectories to land at four different locations in the equatorial region. The speed required to insert the lander into the landing trajectory is mapped onto the parts of the trajectory whose distance to the barycenter is larger than the $\mathrm{L} 2$ distance. $a$ ) shows the landing at $0^{\circ}$ longitude, which faces the L2 point. $b$ - $f$ ) depict trajectories landing at $90^{\circ}, 140^{\circ}, 246.5,244$ and 211 longitudes, respectively.

The deployment analysis shows that some of the lowest energy landing condition also come with lengthy landing operations. However, long landing operations are also of increasing risk due to both the highly perturbed environment and the passive nature of the landing, this issue is briefly explored in the following section. Besides, the continuous availability of personnel during lengthy landing operations may also impact on mission costs, and so be worthy of consideration.

Overall, these results show that even in binary systems with total mass on the order of $10^{12} \mathrm{~kg}$, the mothership will require a deployment subsystem that is able to provide $\mathrm{cm} / \mathrm{s}$ level separation speeds, likely with high accuracy. Recall that the deployment mechanism of Rosetta spacecraft was capable of providing a separation speed between 5 $-50 \mathrm{~cm} / \mathrm{s}$, including an emergency system providing of $17 \mathrm{~cm} / \mathrm{s}$ push to its $\sim 10^{13} \mathrm{~kg}$ target, comet $67 \mathrm{P} / \mathrm{Churyumov}-$ Gerasimenko [28]. More relevant to these results, AIM's deployment mechanism was estimated to provide a range of $0.5-2 \mathrm{~m} / \mathrm{s}$ speed for its CubeSat payloads, although this seemed under assessment initially [29]. At the time of writing, AIM's deployment mechanism, upgraded from ISIPOD deployer, is estimated to provide $2-5 \mathrm{~cm} / \mathrm{s}$ within $+/-1 \mathrm{~cm} / \mathrm{s}$ accuracy [30]. The results provided here seems to be in harmony with this upgraded range of deployment speeds, as the majority of darkest shaded regions $(0.2 \mathrm{~m} / \mathrm{s}$ or lower $)$ in Fig 7 actually correspond to values in this range. Note that AIM's binary target, Didymos, has a total system mass on the order of $\sim 5 \times 10^{11} \mathrm{~kg}[2]$.

\section{Further Considerations on the Landing Opportunities}

Despite the existence of landing opportunities as described above, there are other important questions that would ultimately drive the feasibility of low energy ballistic landing trajectories. This last section broadly explores two critical issues: firstly, the dissipation of energy required at touchdown and, secondly, the robustness, or sensitivity, of these landing solutions to uncertainties of motion. The former is a necessary condition to enable a passive landing, since if the landing were to exhibit the behaviour of elastic collisions, then the resultant motion would be an escape trajectory. The robustness, or sensitivity, is a critical issue for low energy trajectories, since these exhibit highly chaotic behaviours, and may ultimately render some of the lowest energy trajectories as computed earlier unfeasible, 
if sufficient robustness to uncertainties cannot be ensured. However, this section does not intend to provide a comprehensive analysis on these issues, but only some final insight in the prospective usefulness of the landing trajectories, to complement the previous discussion.

\section{A. Bouncing Motion and Coefficient of Restitution}

It is clear that if the local vertical touchdown velocity is simply reverted instantaneously, without any loss of energy, then the resultant trajectory is a take-off trajectory with the same allowed motion that the original landing had. Therefore, some dissipation of energy must occur in order for the spacecraft to, eventually, settle on the surface of the asteroid. Fortunately, energy dissipation is expected to occur naturally as undoubtedly some energy will be lost to heat and deformation during the short touchdown instant, before bouncing occurs. This energy dissipation is here represented by the coefficient of restitution $\varepsilon_{V}$, which defines the ratio between the local vertical velocity $\mathbf{v}_{L V}^{+}$, an instant after the touchdown (+), and the local vertical velocity $\mathbf{v}_{L V}^{-}$, an instant before the touchdown (-).

Fig. 9 summarises the values of the coefficient of restitution $\varepsilon_{V}$, as a function of the landing site, that ensures a sufficient condition to remain permanently in the neighbourhood of the body where the landing has been attempted.

This sufficient condition is computed by estimating a coefficient of restitution $\varepsilon_{V}=\mathbf{v}_{L V J 1}^{+} / \mathbf{v}_{L V}^{-}$, where $\mathbf{v}_{L V J 1}^{+}$represents the local vertical velocity after touchdown such that the Jacobi constant of the bouncing trajectory is that of the L1 equilibrium. This energy limit after the first bounce is chosen so that no escape can possibly occur from the neighbourhood of the asteroid, since zero velocity surfaces will be closed both at L1 and L2 points. As can be observed in Fig. 9, the far side of the secondary (L2 facing) is characterised by $\varepsilon>0.8$, while the highest $\varepsilon_{V}$ in the primary body occurs near the equator within the range of 0.6 to 0.7 , except in some small regions which are artificially affected as a consequence of the constrained landing geometry together with the secondary position. 


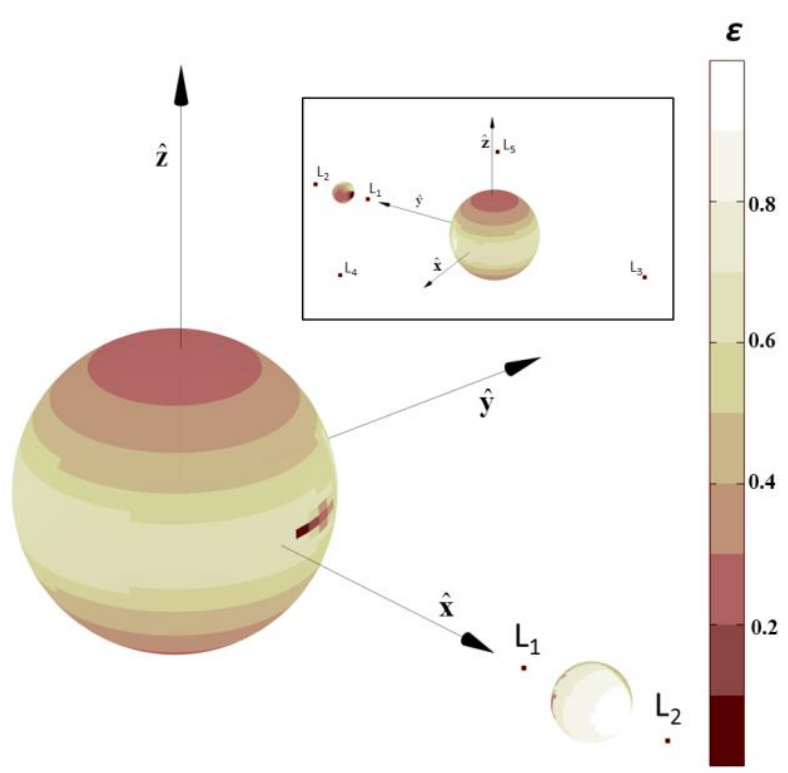

Fig. 9 Required coefficient of restitution $\varepsilon$ to ensure ZVS closed at L1 \& L2 points

Clearly, a reliable estimate of the likely bouncing and settling phase is out of the scope of this paper, since this motion would be highly affected by the local shape and features, such as local slope, boulders, materials, etc [24]. However, the analysis carried out for Fig. 10 indicates that the coefficient of restitution $\varepsilon_{V}=\mathbf{v}_{L V J 1}^{+} / \mathbf{v}_{L V}^{-}$is likely only a lower bound and that landing may also be possible at higher $\varepsilon_{V}$ (i.e, sufficient but not necessary condition). Fig. $10 a$ represents again the coefficient of restitution as $\varepsilon_{V}=\mathbf{v}_{L V J 1}^{+} / \mathbf{v}_{L V}^{-}$on a Mercator projection of the secondary. Fig. $10 b$ instead represents a $\varepsilon_{V}=\mathbf{v}_{L V}^{+} / \mathbf{v}_{L V}^{-}$where $\mathbf{v}_{L V}^{+}$is iteratively computed with a bisection method so that its forward propagation is ensured to remain on the secondary after only two bounces. This means that at the second bounce sufficient energy damping has already occurred so that zero velocity surfaces are closed at L1 and L2 points. The dynamics of bouncing motion are computed here assuming an impulsive rebound approximation as described by Eq.(7-8). Note that, in this case, the coefficient of restitution of the local-horizontal component of the velocity $\varepsilon_{H}$ is assumed 1 , although in any real situation this is likely to be smaller than $\varepsilon_{V}$ [24, 25]. Fig. $10 b$ then demonstrates that by only allowing one complete bounce, whose damping of energy only occurs in the local vertical direction, the region on which $\varepsilon<0.9$ would result on successful landing on the secondary has already grown considerably. 

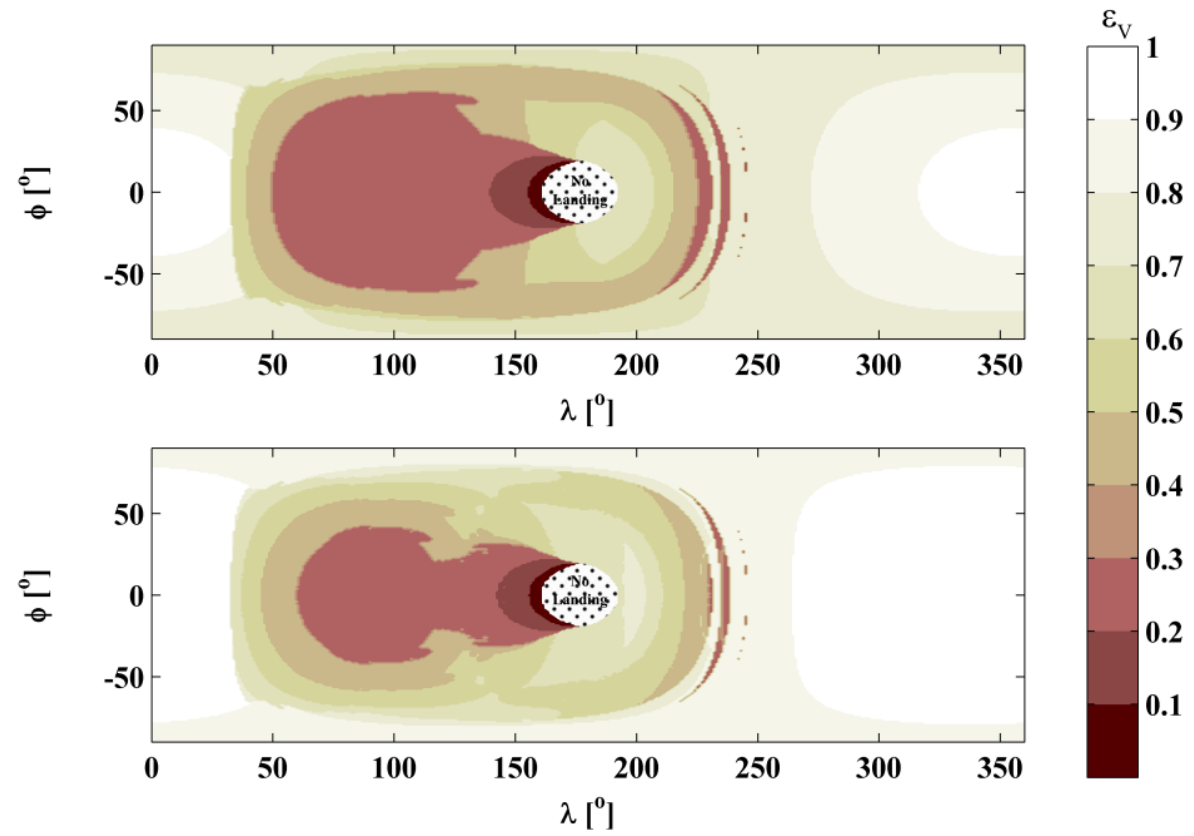

Fig. 10 Coefficient of restitution $\varepsilon_{V}$ required to ensure a passive landing onto the secondary. a) Estimates computed by means of energy consideration (i.e., remaining energy after first bound must be smaller than $\mathrm{C}_{\mathrm{L} 1}$ ). b) Estimates computed by numerically iterating bounces assuming an impulsive rebound model as in Eq.(7-8).

The results shown in Fig. 10 are of course strictly relevant only for a spherical secondary with no surface features, such as boulders, on the surface, which may completely distort the bouncing motion. Nevertheless, these results cast some important insight into the feasibility of ballistic landing onto a binary system, especially when considering that past missions that touched down onto the surface of asteroids or comets, such as Hayabusa and Philae, all measured coefficients of restitution of $\varepsilon<0.85$ [28, 31]. Hence, these results indicate the potential for ballistic landing on some particular regions of the secondary, and so demonstrate the merit of investigating this concept with higher fidelity models.

\section{B. Sensitivity of the Deployment Conditions.}

Inherent to the benefits and advantages of low energy conduits associated with periodic motion near equilibrium points also come the instabilities of motion near these regions [20, 32]. As discussed earlier, many of the landing trajectories analysed here wind around the equilibrium points of the CR3BP, indicating their proximity to periodic motion near these points. Consequently, an understanding of the sensitivities of these landing trajectories to 
uncertainties both due to the strongly perturbed environment and to the errors during the deployment operation is required in order to assess the feasibility of these landing trajectories. This section attempts to gain some final insight into the dynamical sensitivity of the landing trajectories by assessing the dispersion of the landing trajectories subject to errors on the deployment velocity vector. Fig. 11 summarises the results of this analysis.

Fig. $11 a$ shows the $\log 10$ of the spectral norm of the state transition matrix (SSM) $\boldsymbol{\Phi}\left(t, t_{0}\right)$, such that the difference between two neighbouring landing trajectories with initial state at deployment as $\mathbf{s}_{0}$ and $\mathbf{s}_{0}+\delta \mathbf{s}_{0}$ can be estimated at first order as:

$$
\delta \mathbf{s}=\mathbf{\Phi}\left(t, t_{0}\right) \delta \mathbf{s}_{0}
$$

where the SSM $\boldsymbol{\Phi}\left(t, t_{0}\right)$ is numerically integrated as:

$$
\begin{aligned}
& \dot{\boldsymbol{\Phi}}\left(t, t_{0}\right)=\mathbf{A}(t) \boldsymbol{\Phi}\left(t, t_{0}\right) \\
& \mathbf{\Phi}\left(t_{0}, t_{0}\right)=\mathbf{I}
\end{aligned}
$$

and $\mathbf{A}(t)$ is the matrix of partial derivatives of the system, or Jacobian matrix [33]. The spectral norm of the SSM for the landing trajectory provides a very rough understanding on how quickly a neighboring state will diverge from the actual landing trajectory, as discussed by the author in [34].

However, as a figure of merit, the $\log 10$ of the spectral norm of $\boldsymbol{\Phi}\left(t, t_{0}\right)$ only provides some qualitative insight of the regions whose landing trajectories may be more sensitive to uncertainties. Further to Fig. 11a, Fig. $11 b$ complements the analysis by providing the results on a Monte Carlo simulation. Fig. $11 b$ shows the success rate of a 1000 landing sequences where a random $1 \mathrm{~cm} / \mathrm{s}$ error is introduced in the deployment manoeuvre. The $1 \mathrm{~cm} / \mathrm{s}$ error is added as a pseudorandom error in magnitude $\left(\left[\begin{array}{ll}0 & 1\end{array}\right] \mathrm{cm} / \mathrm{s}\right)$, right ascension and declination $\left(\left[\begin{array}{ll}0 & 2 \pi\end{array}\right] \mathrm{rad}\right)$ of the velocity. Due to the addition of error to the nominal trajectory, the touchdown can occur in a different position than the original nominal touchdown position (where the trajectory takes off in backwards propagation), and it does not have to be in local vertical. Note that here it is sufficient for a trajectory to touchdown once on the secondary to be considered successful. It is not the scope of this simulation to assess the viability to successfully land given the velocity uncertainty, but simply to detect potential issues on the sensitivities to errors in the velocity vector at deployment. Indeed, both Fig. $11 a$ and $b$ show that the region directly looking towards the L2 point is extremely sensitive, while adjacent regions seem to be less so. The near equatorial region between 250 and 340 degrees longitude, however, not only appear to be relatively robust to uncertainties in velocity, but also correspond with 
regions where only modest energy damping is required to ensure landing. Finally, note that in Fig. $11(a$ and $b)$ there is a large region where landing is not possible. The region, marked with a diagonal line texture, appears due to the fact that landing in Fig. 11 is required to occur within 10h of the deployment. In these no-landing regions then, it appears that landing would require longer than $10 \mathrm{~h}$.

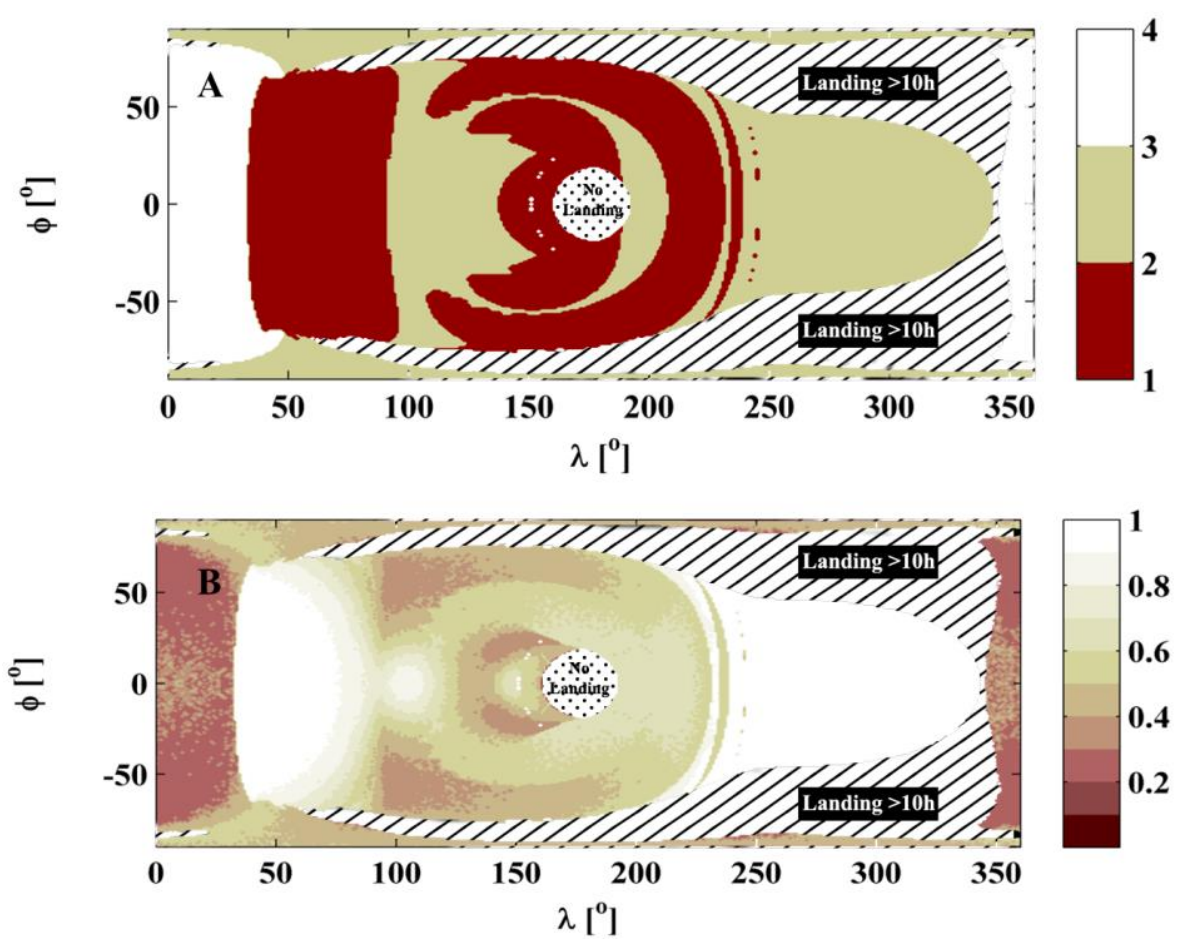

Fig. 11 Sensitivity analysis for deployment conditions. a) $\log 10$ of the spectral norm of the landing trajectory transition matrix. $b$ ) touchdown success rate for a 1000 trials Monte Carlo simulation with added uncertainties in the velocity. Diagonal pattern represents regions where landing is not possible.

It is out of the scope of this paper to provide a comprehensive discussion on the technical viability of these landing trajectories, for which a much higher fidelity characterization of the landing scenario would be required (e.g., deployment errors, gravitational and non-gravitational perturbations, etc.). However, the results presented in Fig. 11, together with the previous analysis, continues to indicate the prospective opportunities for ballistic landing in binary asteroids, particularly, onto the surface of the secondary. Clearly, further analyses are required for specific binary systems, with a much more specific characterization of the dynamical model, its perturbations and the technical limits of the deployment from the exterior region. However, the results shown in this paper clearly indicate the merit of continuing with these analyses further. 


\section{Conclusions}

This paper has investigated the possibility to benefit from the natural dynamics near a binary asteroid as an efficient mean to deliver a lander, or scientific payload, onto its surface. The results show that the dynamics of the landing options onto the surface of the primary are always subject to high energies, as in the sense that the energy of the landing trajectory is far above that of the L4/L5 equilibrium position. However, the options to land onto the secondary, particularly the region directly facing the L2 point, benefit from well-known low energy conduits in the three-body problem.

The scalability of the model used in this paper allows to carry out a simple, yet effective, study of accessible regions and landing/deployment speeds that can easily extend to any binary size. The backwards propagation approach has proved to be a powerful tool to assess deployment options at a variety of locations in the exterior region. The algorithm is flexible, and specific conditions and constraints for the landing trajectories can be easily added. In this regard, a simple, yet robust, method is presented to evaluate the feasibility of natural landing trajectories in binary asteroid systems. The mass parameter of the binary system was defined as a function of the primary-to-secondary ratio, and the results focus on a hypothetical binary system designed as a good representative of the known binaries within the NEA population.

The results show that while landing on the primary may yet put some challenging constraints to the spacecraft, since it would require an effective passive/active landing system, the secondary offers the prospect of simple totally ballistic landing opportunities. This prospect is bound to the condition that the mothership is capable of deploying the lander with sufficient accuracy. However, the analysis carried out here does not allow us to estimate what is the required accuracy, since this would depend on the particular binary asteroid targeted, and much more accurate study should be performed. Nevertheless, it is also clear that, while deployment mechanisms for Philae and MASCOT provide relatively accurate, low speed deployments, current standardized deployment mechanisms (PPOD) for CubeSats do not provide the gentle impulses that would be required to enable passive landing in binary systems. Further improvement on PPOD or a modification on the existing deployment mechanism for bigger landers may be required to accurately deploy CubeSat payloads to binary asteroids.

The landing trajectories computed here represent only the minimum energy landing trajectories for a vertical landing at a given latitude and longitude, at both primary and secondary. Slightly different landing conditions (i.e., 
direction and velocity) may exist, exhibiting much more optimal features, in terms of landing duration and sensitivity to perturbations, at modest increases of coefficient of restitution.

\section{Acknowledgments}

Onur Çelik would like to acknowledge the Erasmus Mundus Master Course in Space Science and Technology SpaceMaster consortium for the valuable scholarship and their help throughout his master studies.

\section{References}

[1] Margot, J. L., Nolan, M. C., Benner, L. a M., Ostro, S. J., Jurgens, R. F., Giorgini, J. D., Campbell, D. B., "Binary asteroids in the near-Earth object population," Science, Vol. 296, No. 5572, 2002, pp. 1445, 1448.

doi: 10.1126/science. 1072094

[2] Cheng, A. F., Atchison, J., Kantsiper, B., Rivkin, A. S., Stickle, A., Reed, C., Ulamec, S., “Asteroid Impact and Deflection Assessment mission," Acta Astronautica, Vol. 115, 2015, pp. 262, 269.

doi: 10.1016/j.actaastro.2015.05.021

[3] Dunham, D., "Implementation of the First Asteroid Landing," Icarus, Vol. 159, No. 2, 2002, pp. 433, 438. doi: 10.1006/icar.2002.6911

[4] Tsuda, Y., Yoshikawa, M., Abe, M., Minamino, H., \& Nakazawa, S., "System design of the hayabusa 2-asteroid sample return mission to 1999 JU3," Acta Astronautica, Vol. 91, 2013, pp. 356, 362.

doi: 10.1016/j.actaastro.2013.06.028

[5] Karatekin, Ö., and the AGEX team, "The Asteroid Geophysical EXplorer (AGEX) to explore Didymos," 5th Interplanetary CubeSat Workshop (iCubeSat), Oxford, 2016.

[6] Tardivel, S., Canalias, E., Deleuze, M., Klesh, A.T., Scheeres, D.J., "Landing MASCOT on asteroid 1999 JU3: solutions for deploying nanosats to small body surfaces," 46th Lunar and Planetary Science Conference, 2015.

[7] Tardivel, S., and Scheeres, D. J., "Ballistic Deployment of Science Packages on Binary Asteroids," Journal of Guidance, Control, and Dynamics, Vol. 36, No. 3, 2013, pp. 700, 709.

doi: $10.2514 / 1.59106$

[8] Tardivel, S., Michel, P., and Scheeres, D. J., "Deployment of a lander on the binary asteroid (175706) 1996 FG3, potential target of the european MarcoPolo-R sample return mission,” Acta Astronautica, Vol. 89, 2013, pp. 60, 70. doi: 10.1016/j.actaastro.2013.03.007

[9] Herrera-Sucarrat, E., Palmer, P. L., and Roberts, R. M., “Asteroid Observation and Landing Trajectories Using Invariant Manifolds,” Journal of Guidance, Control, and Dynamics, Vol. 37, No. 3, 2014, pp. 907, 920. 
doi: $10.2514 / 1.59594$

[10]Sawai, S., Kawaguchi, J., Scheeres, D. J., Yoshikawa, N., and Ogasawara, M., "Development of a Target Marker for Landing on Asteroids," Journal of Spacecraft and Rockets, Vol. 38, No. 4, 2001, pp. 601, 608.

doi: $10.2514 / 2.3723$

[11]Kubota, T., Sawai, S., Hashimoto, T., and Kawaguchi, J., "Collision dynamics of a visual target marker for small-body exploration," Advanced Robotics, Vol. 21, No. 14, 2007, pp. 1635, 1651.

doi: $10.1163 / 156855307782227426$

[12]Pravec, P., and Harris, a. W., "Binary asteroid population,” Icarus, Vol. 190, No. 1, 2007, pp. $250,259$. doi: 10.1016/j.icarus.2007.02.023

[13]Rubincam, D., "Radiative Spin-up and Spin-down of Small Asteroids,” Icarus, Vol. 148, No. 1, 2000, pp. 2, 11. doi: 10.1006/icar.2000.6485

[14]Jacobson, S. A., and Scheeres, D. J., "Dynamics of rotationally fissioned asteroids: Source of observed small asteroid systems," Icarus, Vol. 214, No. 1, 2011, pp. 161, 178.

doi: 10.1016/j.icarus.2011.04.009

[15]Walsh, K., and Richardson, D., "Binary near-Earth asteroid formation: Rubble pile model of tidal disruptions," Icarus, Vol. 180, No. 1, 2006, pp. 201, 216.

doi:10.1016/j.icarus.2005.08.015

[16]Walsh, K. J., Richardson, D. C., and Michel, P., "Rotational breakup as the origin of small binary asteroids," Nature, Vol. 454, No. 7201, 2008, pp. 188, 191.

doi: $10.1038 /$ nature 07078

[17]Ostro, S. J., Margot, J.-L., Benner, L. a M., Giorgini, J. D., Scheeres, D. J., Fahnestock, E. G., Suzuki, S., "Radar imaging of binary near-Earth asteroid (66391) 1999 KW4,” Science, Vol. 314 No. 5803, 2006, pp. 1276, 1280. doi: $10.1126 /$ science. 1133622

[18]Ćuk, M., and Nesvorný, D., “Orbital evolution of small binary asteroids,” Icarus, Vol. 207, No. 2, 2010, pp. $732,743$. doi:10.1016/j.icarus.2009.12.005

[19]Anderson, R. C., Scheeres, D., Chesley, S., and the BASiX Science Team, "Binary Asteroid in-situ Explorer Mission (BASiX): A Mission Concept to Explore a Binary Near-Earth Asteroid System," 45th Lunar and Planetary Science Conference, LPI-USRA, 2014.

[20]Szebehely, V., Theory of Orbits: The Restricted Problem of Three Bodies, Academic Press, 1963.

[21]Ulamec, S. and Biele, J., "Surface Elements and Landing Strategies for Small Bodies Missions - Philae and Beyond," Advances in Space Research, Vol. 44, No. 7, 2009, pp. 847-858. 
doi: http://dx.doi.org/10.1016/j.asr.2009.06.009

[22]Pignatelli, D., "Poly Picosatellite Orbital Deployer Mk .III Rev. E User Guide", The CubeSat Program, California Polytechnic State University, CP-PPODUG-1.0-1, San Luis Obispo, 93407, CA, 2014.

[23]Greenwood, D. T., Principles of Dynamics, 2nd ed., London: Prentice Hall, 1988.

[24]Tardivel, S., Scheeres, D. J., Michel, P., van Wal, S. and Sánchez, P., “Contact Motion on Surface of Asteroid,” Journal of Spacecraft and Rockets, Vol. 51, No. 6, 2014, pp. 1857-1871.

doi: 10.2514/1.A32939

[25]Biele, J., Ulamec, S., Maibaum, M., Roll, R., Witte, L., Jurado, E., Muñoz, P., Arnold, W., Auster, H.-U., Casas, C., Faber, C., Fantinati, C., Finke, F., Fischer, H.-H., Geurts, K., Güttler, C., Heinisch, P., Herique, A., Hviid, S., Kargl, G., Knapmeyer, M., Knollenberg, J., Kofman, W., Kömle, N., Kührt, E., Lommatsch, V., Mottola, S., Pardo de Santayana, R., Remetean, E., Scholten, F., Seidensticker, K. J., Sierks, H. and Spohn, T., “The Landing(S) of Philae and Inferences About Comet Surface Mechanical Properties,” Science, Vol. 349, No. 6247, 2015.

doi: $10.1126 /$ science.aaa9816

[26]Ren, Y., and Shan, J., “A novel algorithm for generating libration point orbits about the collinear points," Celestial Mechanics and Dynamical Astronomy, Vol. 120, No. 1, 2014, pp. 57, 75. doi: 10.1007/s10569-014-9560-9

[27]Olikara, Z. P., Gomez, G., and Masdemot, J. J., "End-Of-Life Disposal of Libration Point Orbit Spacecraft," 64rd International Astronautical Congress, Beijing, 2013.

[28]Ulamec, S., Fantinati, C., Maibaum, M., Geurts, K., Biele, J., Jansen, S., and O’Rourke, L., "Rosetta Lander - Landing and operations on comet 67P/Churyumov-Gerasimenko,” Acta Astronautica, In Press.

doi: 10.1016/j.actaastro.2015.11.029

[29]Carnelli, I., Galvez, A. and Walker, R., "Science by Cubes: opportunities to increase AIM science return," 4th Interplanetary CubeSat Workshop (iCubeSat), London, 2015.

[30]Walker, R., Binns, D., Carnelli, I., Kueppers, M., and Galvez, A., "CubeSat Opportunity Payload Intersatellite Network Sensors (COPINS) on the ESA Asteroid Impact Mission (AIM)," 5th Interplanetary CubeSat Workshop (iCubeSat), Oxford, 2016

[31]Yano, H., Kubota, T., Miyamoto, H., Okada, T., Scheeres, D., Takagi, Y., Yoshida, K., Abe, M., Abe, S. and Barnouin-Jha, O., "Touchdown of the Hayabusa Spacecraft at the Muses Sea on Itokawa," Science, Vol. 312, No. 5778, 2006, pp. 13501353

doi: $10.1126 /$ science.1126164 
[32]Gómez, G., Jorba, À., Masdemont, J.J., Simó, C., "Study of the transfer from the Earth to a halo orbit around the equilibrium point L1", Celest Mech Dyn Astr, 56 (1993) 541-562. doi: 10.1007/BF00696185.

[33]Vallado, D.A., Fundamentals of Astrodynamics and Applications, 3rd Edition ed., Microcosm Press/Kluwer Academic Publishers, El Segundo, California, 2007.

[34]Ceriotti, M., and Sanchez, J. P., "Control of asteroid retrieval trajectories to libration point orbits", Acta Astronautica, 2016, in press, doi:10.1016/j.actaastro.2016.03.037. 
2017-03-29

\section{Opportunities for ballistic soft landing in binary asteroids}

Çelik, Onur

American Institute of Aeronautics and Astronautics

Onur Çelik and Joan Pau Sánchez. Opportunities for Ballistic Soft Landing in Binary Asteroids, Journal of Guidance, Control, and Dynamics, Vol. 40, No. 6 (2017), pp1390-1402.

http://dx.doi.org/10.2514/1.G002181

Downloaded from Cranfield Library Services E-Repository 